\title{
Slug bubble growth and dissolution by solute exchange
}

\author{
Daniël P. Faasen $\odot,{ }^{*}$ Devaraj van der Meer $\odot,^{\dagger}$ Detlef Lohse $\odot,{ }^{\ddagger}$ and Pablo Peñas $\overbrace{}^{\S}$ \\ Physics of Fluids Group, Faculty of Science and Technology, \\ University of Twente, P.O. Box 217, 7500 AE Enschede, The Netherlands
}

(Received 9 June 2021; accepted 16 November 2021; published 29 November 2021)

\begin{abstract}
In many environmental and industrial applications, the mass transfer of gases in liquid solvents is a fundamental process during the generation of bubbles for specific purposes or, vice versa, the removal of entrapped bubbles. We address the growth dynamics of a trapped slug bubble in a vertical glass cylinder under a water barrier. In the studied process, the ambient air atmosphere is replaced by a $\mathrm{CO}_{2}$ atmosphere at the same or higher pressure. The asymmetric exchange of the gaseous solutes between the $\mathrm{CO}_{2}$-rich water barrier and the air-rich bubble always results in net bubble growth. We refer to this process as solute exchange. The dominant transport of $\mathrm{CO}_{2}$ across the water barrier is driven by a combination of diffusion and convective dissolution. The experimental results are compared to and explained with a simple numerical model, with which the underlying mass transport processes are quantified. Analytical solutions that accurately predict the bubble growth dynamics are subsequently derived. The effect of convective dissolution across the water layer is treated as a reduction of the effective diffusion length, in accordance with the mass transfer scaling observed in laminar or natural convection. Finally, the binary water-bubble system is extended to a ternary water-bubble-alkane system. It is found that the alkane ( $n$-hexadecane) layer bestows a buffering (hindering) effect on bubble growth and dissolution. The resulting growth dynamics and underlying fluxes are characterized theoretically.
\end{abstract}

DOI: 10.1103/PhysRevFluids.6.113501

\section{INTRODUCTION}

The dissolution of carbon dioxide gas and its subsequent transport through liquid layers plays an important role in many industrial and biological applications. Examples include enhanced oil recovery [1], $\mathrm{CO}_{2}$ sequestration operations in porous media [2], and gas exchange in the respiratory system [3]. In microfluidics, segmented flows of alternating $\mathrm{CO}_{2}$ gas and liquid plugs are frequently employed to study solvent absorption properties and chemical reaction kinetics [4-6]. In fact, the controlled dissolution of $\mathrm{CO}_{2}$ bubbles constitutes a crucial step in the microfluidic generation of encapsulated microbubbles for ultrasound imaging in medical diagnostics and targeted drug delivery $[7,8]$.

Bubble formation, however, can have detrimental effects on microfluidic devices such as blocking the liquid flow $[9,10]$. Bubbles covering the electrode surface of electrochemical devices hinder gas-evolution reactions, thereby reducing the efficiency of the cell [11-15]. Similarly, bubble formation during inkjet printing can completely disrupt the printing process [16]. Therefore, thorough

\footnotetext{
*d.p.faasen@utwente.nl

d.vandermeer@utwente.nl

$\doteqdot$ d.lohse@utwente.nl

§p.penaslopez@utwente.nl
} 
(a)

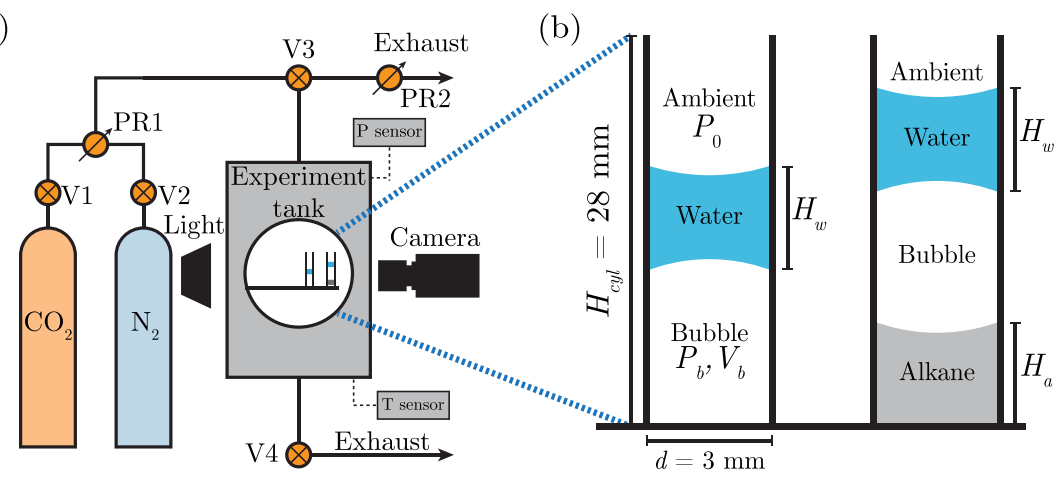

FIG. 1. (a) Schematic overview of the experimental setup. (b) Sketch of the cylinders containing the water-bubble and water-bubble-alkane systems. These are placed inside the chamber, which is subsequently pressurized with $\mathrm{CO}_{2}$ gas.

understanding of the solubility and transport of dissolved gas is clearly of benefit, not only upon encountering these complications, but also in an overwhelming number of applications concerning gas or vapor bubbles [17].

Multiple solutions for unwanted bubble removal exist, ranging from passive removal such as bubble traps, to active removal by increasing the driving pressure in order to increase the gas solubility and induce dissolution [9]. However, when performing the latter, one should take note of the gas composition inside the trapped bubble and surrounding liquid, as pressurizing with a different gas species may very well cause the bubble to grow instead of dissolve.

This is precisely the focus of our work. We investigate the mass transfer experienced by a single slug air bubble trapped in a vertical glass cylinder beneath a thin layer of water which separates the bubble from the ambient gas, as shown in Fig. 1. We replace the ambient air atmosphere with a $\mathrm{CO}_{2}$ atmosphere at the same or higher pressure, and study the subsequent growth dynamics of the bubble. Its growth is driven by the asymmetric exchange of gaseous solutes in the water layer $\left(\mathrm{CO}_{2}\right)$ with those in the trapped bubble (air). We therefore refer to this driving mechanism as solute exchange, where by replacing the gaseous solutes in a liquid solvent one is able to promote the growth (or dissolution) of the bubbles found therein. Solute exchange is fundamentally different from the solvent exchange mechanism used to generate microbubbles by replacing the liquid solvents $[18,19]$.

In a nutshell, our aim is to investigate the growth dynamics of a trapped slug bubble due to a solute exchange process induced by changes in the outer ambient gas composition. It will be seen that the bubble growth rate is in fact supradiffusive due to the onset of $\mathrm{CO}_{2}$ dissolution-driven convection across the vertical water layer above the bubble. Convective dissolution or growth is indeed encountered in many relevant applications, such as $\mathrm{CO}_{2}$ capture and storage in saline aquifers [2], buoyancy-generating chemical reactions [20,21], droplet dissolution [22,23], and bubble growth [24]. In essence, our system embodies a mass-transport variant of the classical problem of RayleighBénard convection [25] in a cylindrical cell. With this in mind, we devote significant effort to the quantification, both experimentally and theoretically, of the effect of convective dissolution on the bubble growth dynamics. Finally, the binary water-bubble system is extended to a ternary waterbubble- $n$-hexadecane system. Bubble growth dynamics will be seen to be hindered by the presence of the buffering alkane layer. Ternary configurations are of considerable relevance in gas-liquidliquid three-phase microreactors [26]. The inclusion of oxygenated oil layers has also been shown to enhance bacterial growth in bioreactors [27], owing to the well-known fact that oils have higher gas solubilities than water [28-30].

This paper is structured as follows. The experimental setup and procedure are described in Sec. II. Section III offers a qualitative description of the solute exchange mechanism. Section IV presents quantitative results for the aforementioned binary water-bubble system. We propose a 
simple theoretical framework which adequately describes the bubble growth dynamics driven by (convection-enhanced) solute exchange. Experiments are compared to a numerical model and analytical solutions. The proposed dependence of the Sherwood number on the Rayleigh number across the water layer is compared to all binary and ternary experiments in Sec. V. In Sec. VI the ternary water-bubble-alkane system is investigated in greater detail, and the buffering effect of the $n$-hexadecane layer is characterized. The paper ends with a summary of the main findings and an outlook in Sec. VII.

\section{EXPERIMENTAL SETUP AND PROCEDURE FOR SOLUTE EXCHANGE}

A schematic overview of our experimental setup is shown in Fig. 1(a). The experiments are conducted inside a sealed chamber which can be pressurized with either $\mathrm{CO}_{2}$ or $\mathrm{N}_{2}$ gas. The inlet pressure is adjusted using pressure regulator PR1, whereas a second pressure regulator, PR2, grants additional control of the ambient pressure $P_{0}$ inside the tank, where $1 \leqslant P_{0} \leqslant 4$ bars. A temperature and a pressure sensor monitor the temperature $T_{0}$ and pressure $P_{0}$ in time. Extensive details on the chamber and pressure control system can be found elsewhere [31].

Two borosilicate glass (Duran) cylinders $(28 \mathrm{~mm}$ in length, inner diameter $d=3.0 \mathrm{~mm}$, outer diameter of $5.0 \mathrm{~mm}$ ) are attached on one end to a silicon wafer plate using Loctite 4305 (Farnell), while the remaining end is left open. Before use, the cylinders are rinsed with ethanol (Boom, technical grade) followed by Milli-Q water (resistivity $=18.2 \mathrm{M} \Omega \mathrm{cm}$ ) and finally dried in a nitrogen stream.

The cylinders host either a water-bubble or a water-bubble-alkane configuration, as depicted in Fig. 1(b). The water-bubble system is prepared by injecting a slug bubble using an air-filled syringe between the plate wall and a previously deposited Milli-Q water layer of height $H_{w}=2.5-6.0 \mathrm{~mm}$. The bubble is held in place due to a stable balance between the weight of the water barrier and a combination of the surface tension of the water-bubble interface and to a lesser extent the differences in gas pressures. This force balance persists during the experiments, given that the surface tension of $\mathrm{CO}_{2}$ on water is almost the same as the surface tension of air on water under our experimental conditions [32]. For the water-bubble-alkane configuration, the bubble is similarly injected between a $n$-hexadecane layer (VWR, 99\% purity) of height of $H_{a} \approx 3.0 \mathrm{~mm}$ and the water layer, whose $H_{w}$ was adequately chosen from within the same range of lengths as in the binary system for fair comparison. The selection of bubble volumes will be discussed shortly.

The experimental procedure is best illustrated with reference to a typical experiment shown in Fig. 2. The prepared cylinders are placed inside the experimental tank. The inlet pressure is set to 1.0 bar with valve V3 remaining closed. At time $t=0$, the camera (Photron FASTCAM Nova s12, $2 \mathrm{fps}$ ) and the pressure and temperature sensors (2 acquisitions per second) start recording. At this point, the water-bubble and water-bubble-alkane systems will be in the equilibrium state [Fig. 2(a)]. The volume of any particular bubble, $V_{b}(t)$, is initially at equilibrium: $V_{b}(0) \equiv V_{\text {eq }}$. At time $t_{f-\text { start }}=5 \mathrm{~s}$, valves V3 and V4 [see Fig. 1(a)] are opened and the system is flushed with $\mathrm{CO}_{2}$ gas in order to fully replace the ambient air in the experimental chamber. This corresponds to the "flush" stage in Fig. 2(e), which plots the pressure inside the experimental chamber, $P_{0}(t)$, during the initial minutes of that particular experiment. After flushing for $60 \mathrm{~s}$, valve V4 is closed and $P_{0}$ is set to the pressure level of the "experiment" stage, e.g., $P_{0}=2.0$ bars for the experiment in Fig. 2. The time $t_{f}$ [typically $75 \mathrm{~s}$, see Fig. 2(e)] importantly refers to the time immediately after the flushing and compression stages have been completed and marks the start of the "experiment" stage. The bubble volume at this point [cf. Fig. 2(b)] is coherently denoted by $V_{f} \equiv V_{b}\left(t_{f}\right)$. Thereafter, despite pressurization, both bubbles experience continuous growth-counterintuitive perhaps-as a consequence of solute exchange. The growth is showcased by snapshots in Figs. 2(b)-(d) and its dynamics is quantified in the $V_{b}(t)$ plot in Fig. 2(f). At the end of the experiment ( $1 \mathrm{~h}$ in this case), valves V1-V3 are closed and V4 is opened in order to depressurize the system. The bubble volumes are extracted from the images using an in-house developed MATLAB script. 
(a)

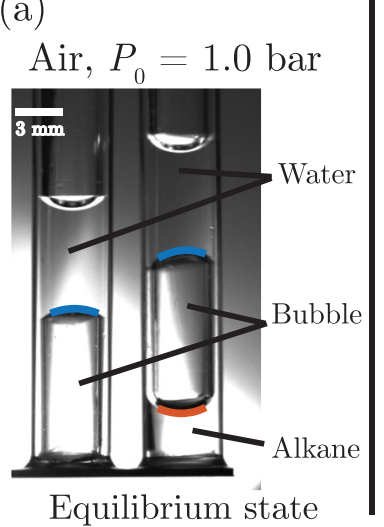

(b)

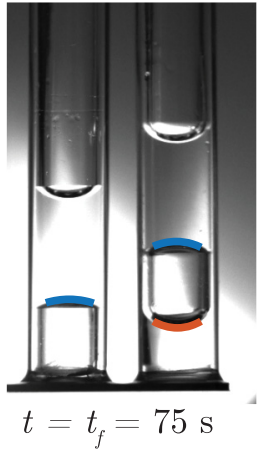

(c)

$\mathrm{CO}_{2}, P_{0}=2.0$ bar

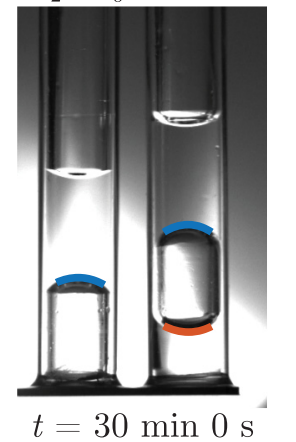

(d)

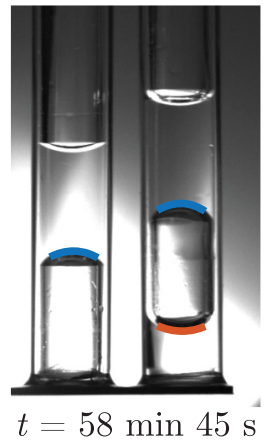

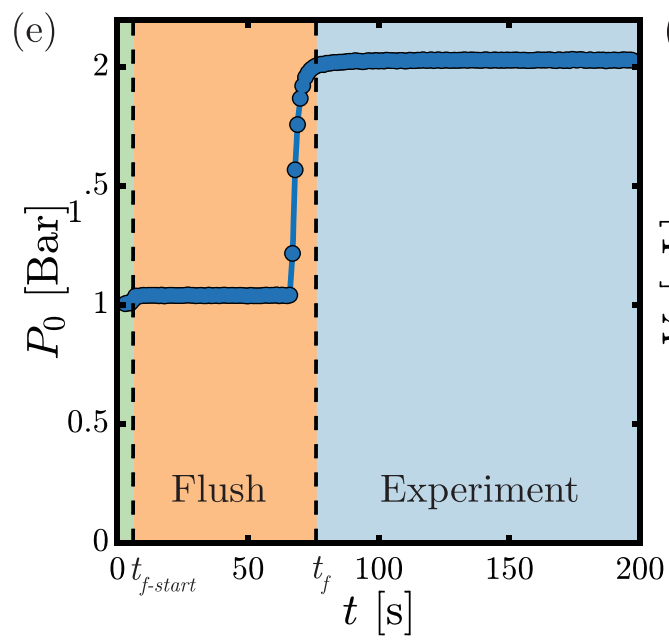

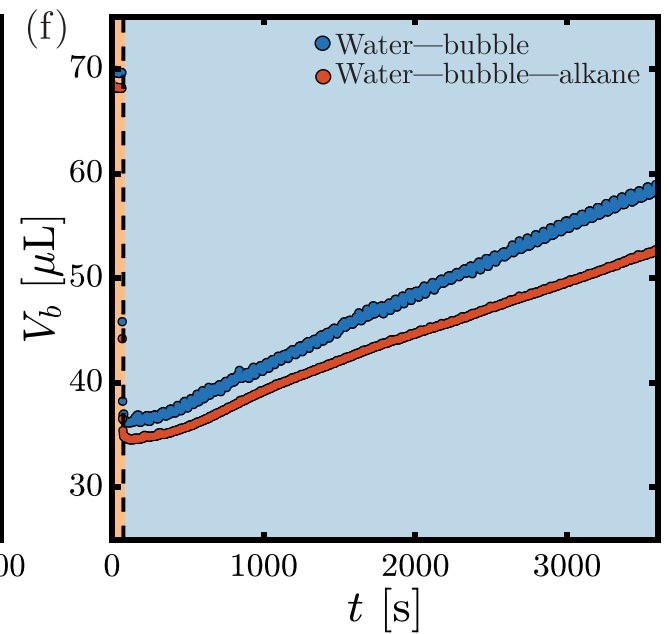

FIG. 2. A typical experiment involving a binary and ternary system. Experimental snapshots at (a) $t=$ 0 , equilibrium state, (b) $t=t_{f}$, immediately after $\mathrm{CO}_{2}$ flushing and pressurization, (c) halfway through the experiment, and (d) at the end of the experiment. The blue line indicates the bubble-water interface detected by the contour detection algorithm. Similarly, in the right cylinder with the alkane, the red line indicates the detected bubble-alkane interface. (e) Time evolution of the pressure in the tank during the first $200 \mathrm{~s}$ of the experiment. Before $t_{f-\text { start }}$ (green stage) the tank is filled with ambient air. Between $t_{f-\text { start }}$ and $t_{f}$ (orange stage) the tank is flushed with $\mathrm{CO}_{2}$ and the bubbles are compressed. (f) Evolution in time of the bubble volume for the two systems.

Several experiments similar to the one just described were performed for binary and ternary systems, or a combination thereof, comprising either a single-system (one-cylinder) experiment or a two-system (two-cylinder) experiment like the one shown in Fig. 2. The pressure level $P_{0}\left(t>t_{f}\right)$ ranged from 1 bar (no compression after flushing) up to 4 bars, with running times of typically 1 or $2 \mathrm{~h}$. The initial bubble volumes considered correspond to (postpressurization) volumes of $25<V_{f}<$ $45 \mu \mathrm{L}(3.5-6.4 \mathrm{~mm}$ in height). The ambient temperature remained fairly constant at approximately $T_{0}=295 \mathrm{~K}$ for every experiment.

It should be stressed that when pressurizing the chamber above the atmospheric pressure $\left[P_{0}(t>\right.$ $\left.t_{f}\right)>1.0 \mathrm{bar}$ ], the bubble volume compresses from $V_{\mathrm{eq}}$ to $V_{f}$ accordingly [cf. Figs. 2(a) and 2(b)], as established by Boyle's law. Moreover, the movement of the contact lines during compression also alters the shape of the bubble menisci, which may even take an additional few seconds to reform and settle. In some cases, we were unable to capture $V_{\text {eq }}$ with sufficient accuracy due to optical 
TABLE I. Henry's constants and diffusion coefficients of the gases in water (superscript $w$ ) and $n$ hexadecane (superscript $a$ ) at $293.0 \mathrm{~K}$ and 1.0 bar. The molar-averaged $S_{\text {air }}^{w}$ can be estimated as $9.02 \times 10^{-6}$ $\mathrm{mol} / \mathrm{m}^{3} \mathrm{~Pa}$.

\begin{tabular}{llccc}
\hline \hline$j$ & $S_{j}^{w}\left(\mathrm{~mol} / \mathrm{m}^{3} \mathrm{~Pa}\right)$ & $S_{j}^{a}\left(\mathrm{~mol} / \mathrm{m}^{3} \mathrm{~Pa}\right)$ & $D_{j}^{w}\left(\mathrm{~m}^{2} / \mathrm{s}\right)$ & $D_{j}^{a}\left(\mathrm{~m}^{2} / \mathrm{s}\right)$ \\
\hline $\mathrm{CO}_{2}$ & $3.79 \times 10^{-4}[28]$ & $4.06 \times 10^{-4}[29]$ & $1.76 \times 10^{-9}[33]$ & $2.20 \times 10^{-9}[34]$ \\
$\mathrm{O}_{2}$ & $1.32 \times 10^{-5}[28]$ & $6.45 \times 10^{-5}[30]$ & $2.03 \times 10^{-9}[35]$ & $2.49 \times 10^{-9} \mathrm{a}[36]$ \\
$\mathrm{N}_{2}$ & $6.89 \times 10^{-6}[28]$ & $4.61 \times 10^{-5}[30]$ & $2.00 \times 10^{-9}[37]$ & $2.49 \times 10^{-9} \mathrm{~b}$ \\
\hline \hline
\end{tabular}

a Only available at $294.0 \mathrm{~K}$.

${ }^{\mathrm{b}}$ Approximated value based on the diffusion coefficient of oxygen in $n$-hexadecane.

limitations. For these reasons, the reference bubble volume is taken to be $V_{f}$ instead of $V_{\text {eq }}$. This decision is justified provided that any changes in the molar gas contents in the bubble (initially air) remain negligible during $t<t_{f}$, i.e., under the assumption that there is insignificant mass transfer to or from the bubble up to that point. The experiments conducted at $P_{0}\left(t>t_{f}\right)=1.0 \mathrm{bar}$ (devoid of pressurization) fully support the above assumption: $V_{f}$ was seen to effectively remain unchanged from $V_{\text {eq. }}$.

\section{SOLUTE EXCHANGE MECHANISM}

The replacement of the air atmosphere inside the experimental chamber by $\mathrm{CO}_{2}$ gas causes the latter to dissolve into the water layer, where a $\mathrm{CO}_{2}$-concentration boundary layer naturally develops. Hence, $\mathrm{CO}_{2}$ is transported down towards the water-bubble interface, where it exsolves into the bubble. The steady-state diffusive influx into the bubble (in the absence of convection) can be estimated noting that the concentration of dissolved $\mathrm{CO}_{2}$ at the water-bubble interface is initially close to zero, whereas at the water-ambient interface it is $S_{\mathrm{CO}_{2}}^{w} P_{0}$, where $S_{\mathrm{CO}_{2}}^{w}$ denotes the Henry coefficient of $\mathrm{CO}_{2}$ in water. The flux estimate is therefore $S_{\mathrm{CO}_{2}}^{w} P_{0} / H_{w}$, where $H_{w}$ is the height of the water column (see Fig. 1).

At the same time, an air concentration gradient forms across the water layer, sustaining a diffusive flux in the opposite direction, i.e., from the (air-rich) bubble to the (air-depleted) $\mathrm{CO}_{2}$ atmosphere. The corresponding diffusive outflux can be estimated in the same manner as $S_{\text {air }}^{w} P_{0} / H_{w}$, where $S_{\text {air }}^{w}$ denotes the molar-averaged Henry coefficient of the air mixture in water. The high solubility of $\mathrm{CO}_{2}$ in water (cf. the solubilities listed in Table I) implies that the $\mathrm{CO}_{2}$ influx is stronger than the air outflux by a factor of $S_{\mathrm{CO}_{2}}^{w} / S_{\text {air }}^{w} \approx 42$. This clear disparity in the magnitude between both solute exchange fluxes results in net bubble growth as seen in Fig. 2.

The time required for diffusion to develop a linear $\mathrm{CO}_{2}$-concentration profile across the full height of the water layer can be estimated as $\sim 0.4 H_{w}^{2} / D_{\mathrm{CO}_{2}}^{w}$ from simple theoretical considerations [see Appendix A, Fig. 10(a)], where $D_{\mathrm{CO}_{2}}^{w}$ denotes the diffusivity of $\mathrm{CO}_{2}$ in water. For the experiment portrayed in Fig. 2 where $H_{w}=3 \mathrm{~mm}$, the bubbles would require an initial transient period of $\approx 2000 \mathrm{~s}$ before the maximum quasisteady growth rate is attained. However, Fig. 2(f) clearly shows that the transient period is substantially smaller, approximately $300 \mathrm{~s}$. Thus, without further calculation, it is evident that convective dissolution must have a dominant influence on the bubble growth. Its effect will be unraveled next in Sec. IV.

Finally, Fig. 2(f) also reveals that the presence of the $n$-hexadecane layer slows down bubble growth. Essentially, part of the $\mathrm{CO}_{2}$ accumulating into the bubble is redissolving into the hexadecane layer, given that it is initially depleted of $\mathrm{CO}_{2}$. The stabilizing or buffering effect of the alkane layer on the bubble growth (and dissolution) dynamics will be discussed in detail in Sec. VI. 
(a)
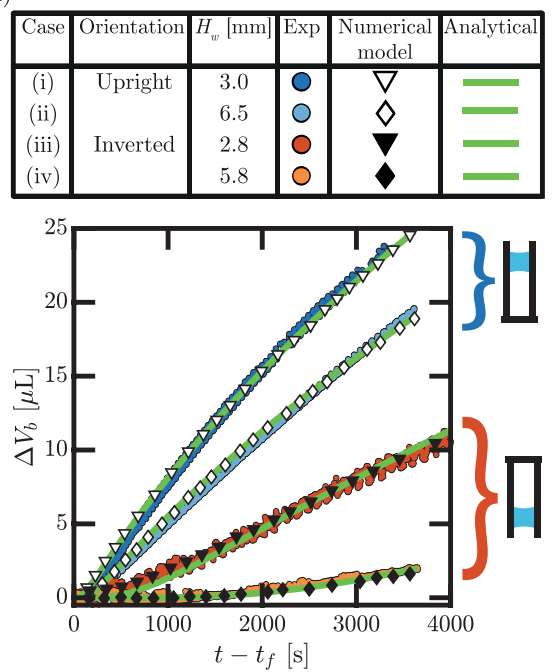

(b)

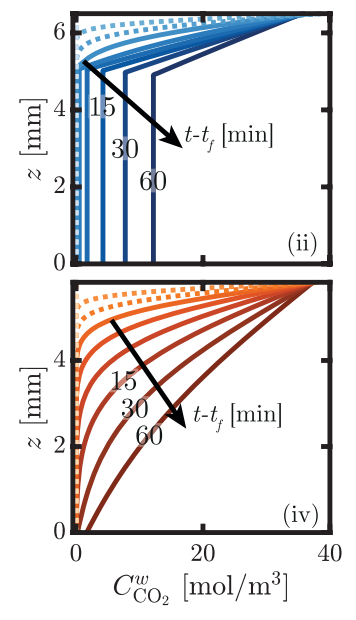

(c)

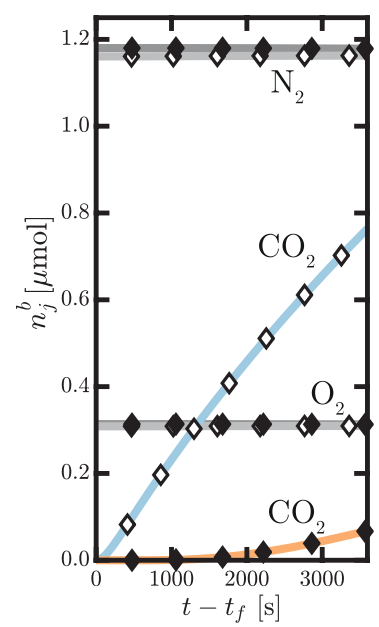

FIG. 3. Experimental and theoretical results for water-bubble systems in an upright configuration [cases (i) and (ii)] and an inverted configuration [cases (iii) and (iv)] for two distinct water layer heights; the experiment pressure level is $P_{0}\left(t>t_{f}\right)=1$ bar and bubble volumes are initially $V_{f}=35 \mu \mathrm{L}$. (a) Bubble growth dynamics $\left(\Delta V_{b} \equiv V_{b}(t)-V_{f}\right)$, according to experiment (circles), numerical model (black and white markers), and analytical solutions (green lines); the latter are given by Eq. (13) for cases (iii) and (iv) and Eq. (17) for cases (i) and (ii). The onset of convective dissolution for upright cases (i, ii) is evident. (b) Time evolution of the $\mathrm{CO}_{2}$ concentration profiles in the water layer according to the model for the convective dissolution case (ii) and the pure diffusion case (iv). The uniform profile outside the diffusion boundary layer in case (ii) illustrates the model assumption of instant convective transport. (c) Time evolution of the gas composition in the bubble according to the model for upright case (ii) (white markers and lighter curves), and inverted case (iv) (black markers and darker curves).

\section{SOLUTE EXCHANGE IN THE WATER-BUBBLE SYSTEM}

In this section, we focus solely on the experiments concerning the binary (bubble-water) configuration. We present a theoretical framework for diffusion-driven solute exchange with the additional effect of dissolution-driven convection. The insight gained from a simple numerical model allows us to derive analytical solutions that accurately describe the dynamics of bubble growth.

\section{A. Effect of convective dissolution}

We begin analyzing a series of single-cylinder water-bubble experiments concerning a bubble of equilibrium volume $V_{\mathrm{eq}}=35 \mu \mathrm{L}$ capped by a water layer with a thickness of either $H_{w} \approx 3 \mathrm{~mm}$ or $H_{w} \approx 6 \mathrm{~mm}$. The prepared cylinders were placed in the experimental chamber, positioned either upright or inverted (rotated $180^{\circ}$ ), as illustrated in Fig. 3(a). Subsequently, the air inside the chamber was replaced by a $\mathrm{CO}_{2}$ atmosphere at $P_{0}\left(t>t_{f}\right)=1$ bar. Therefore, the bubble volume at the start of the "experiment" stage, $V_{f} \equiv V_{b}\left(t_{f}\right)$, is equal to $V_{\text {eq }}$.

The increase in bubble volume with time, $\Delta V_{b}(t) \equiv V_{b}(t)-V_{f}$, is shown for both the upright cases (i and ii) and inverted cases (iii and iv) in Fig. 3(a). The bubbles in the upright configuration are seen to grow much faster than when inverted under otherwise the same conditions. As a consequence, this difference should be attributed to the effect of convective dissolution. In the upright cases, the dense $\mathrm{CO}_{2}$-rich boundary layer that forms at the top of the water phase becomes gravitationally unstable. This gives rise to dissolution-driven convection in the form of $\mathrm{CO}_{2}$-rich viscous fingers or plumes that propagate downwards at relatively high speeds [2,38-41]. In contrast, 
the $\mathrm{CO}_{2}$-water mixture is stably stratified when the cylinder is placed inverted and therefore $\mathrm{CO}_{2}$ can only be transported up the water layer by diffusion.

In the absence of convection, the diffusive transport of the solute exchange (and consequently the bubble growth dynamics) is governed by the one-dimensional diffusion equation for the molar concentration,

$$
\frac{\partial C_{j}^{w}}{\partial t}=D_{j}^{w} \frac{\partial^{2} C_{j}^{w}}{\partial z^{2}}
$$

across the water layer $\left(0<z<H_{w}\right)$ for three gas species: $j=\mathrm{CO}_{2}, j=\mathrm{O}_{2}$, and $j=N_{2}$, where the latter two represent the air mixture. In doing so, we neglect the three-dimensional curvature of the bubble meniscus, whose height is indeed small in comparison with $H_{w}$ [cf. Figs. 2(a)-(d)]. The diffusivity of gas $j$ in water is denoted by $D_{j}^{w}$. The diffusive fluxes across the water-bubble interface on $z=0$ are therefore $J_{j}^{w b}=-D_{j}^{w} \partial_{z} C_{j}^{w}(0, t)$. The ambient pressure $P_{0}(t)$ and the partial pressures in the ambient are prescribed at all times, and these can be interpolated and inferred from the experimental pressure data. The pressure inside the bubble $P_{b}(t)$ is assumed equal to $P_{0}(t)$ at all times, given that the capillary overpressure in the bubble is negligible: $4 \sigma / d \ll P_{0}$, with $\sigma$ as the surface tension. The partial gas pressures in the ambient and the bubble set the interfacial concentrations (boundary conditions) of Eq. (1) as established by Henry's law (see Appendix B). The bubble contents are treated as a mixture of ideal gases; $P_{0} V_{0}=n_{b} R T_{0}$, where $R$ is the universal gas constant and $n_{b}=n_{\mathrm{CO}_{2}}^{b}+n_{\mathrm{N}_{2}}^{b}+n_{\mathrm{O}_{2}}^{b}$ the number of moles of gas in the bubble [neglecting other gases which are present in small amounts $(<1 \%)]$. Finally mass conservation, $J_{j}^{w b} \pi d^{2} / 4=\dot{n}_{j}^{b}$, yields the volume growth rate:

$$
\dot{V}_{b}=\frac{\pi d^{2}}{4} \frac{R T_{0}}{P_{0}} \sum_{j} J_{j}^{w b} .
$$

The above system of Eqs. (1) and (2) is solved and integrated numerically by means of a finite-differences scheme. A complete description of the model equations, initial conditions, and numerical implementation can be found in Appendix A. The resulting time evolution $V_{b}(t)$ is plotted by the black markers in Fig. 3(a) for cases (iii) and (iv). Good agreement is found between these and experiments.

It should be pointed out that we neglect the influence of water vapor (evaporation) on the bubble growth, despite the likely fact that the bubble is saturated with water vapor. This is justified since the water vapor pressure is small, $P_{w}^{\mathrm{sat}} / P_{0}<2 \%$, and its inclusion in the theoretical framework would add little insight at the cost of added complexity. Water evaporation, unlike solute exchange, is not a driving force for bubble growth. Instead, the role of evaporation is merely to ensure that the bubble remains saturated with water vapor at all times. It can be shown that evaporation fluxes amplify $\dot{V}_{b}(t)$ by a factor $\left(1-P_{w}^{\text {sat }} / P_{0}\right)^{-1}$; the resulting difference still remains within the experimental uncertainty of $V_{b}$.

Next, we incorporate the effect of convective dissolution into the solute exchange diffusive framework established so far. Convective transport of $\mathrm{CO}_{2}$ across the water layer is characterized by the instantaneous Rayleigh and Sherwood numbers,

$$
\mathrm{Ra}_{w}(t) \equiv \frac{\lambda \mathrm{CO}_{2} \Delta C_{\mathrm{CO}_{2}}^{w}(t) g H_{w}^{3}}{v_{w} D_{\mathrm{CO}_{2}}^{w}}, \quad \mathrm{Sh}_{w}(t) \equiv \frac{J_{\mathrm{CO}_{2}}^{w b}(t) H_{w}}{D_{\mathrm{CO}_{2}}^{w} \Delta C_{\mathrm{CO}_{2}}^{w}(t)},
$$

where $g$ is the acceleration due to gravity, $v_{w}$ the kinematic viscosity of water, and $\lambda_{\mathrm{CO}_{2}}^{w} \approx$ $8.2 \mathrm{~cm}^{3} / \mathrm{mol}$ the solutal expansion coefficient of $\mathrm{CO}_{2}$ in water at $T_{0}=295 \mathrm{~K}[42,43]$. The instantaneous concentration difference across the water layer is established by the partial pressure difference of $\mathrm{CO}_{2}$ gas that exists between the ambient and the bubble: $\Delta C_{\mathrm{CO}_{2}}^{w}(t)=S_{\mathrm{CO}_{2}}^{w}\left[P_{\mathrm{CO}_{2}}^{0}(t)-P_{\mathrm{CO}_{2}}^{b}(t)\right]$.

In view of the modest aspect ratios of the water column considered in our experiments $(0.45<$ $d / H_{w}<1.3$ ), we have adopted $H_{w}$ as the relevant length scale, in agreement with the classical 
definition of the Rayleigh number in the field of Rayleigh-Bénard convection [44,45]. Here it is worth noting that in contrast to the above choice, convective dissolution studies often employ porous Rayleigh and Sherwood numbers based on the permeability of the porous medium $[2,38,40,46]$. There, the permeability scales as $d^{2}$, namely, the square of the highly restrictive pore size or the Hele-Shaw cell thickness.

The characteristic Rayleigh number can be readily estimated from Eq. (3) inserting $H_{w}=$ $3.0 \mathrm{~mm}, P_{\mathrm{CO}_{2}}^{0}-P_{\mathrm{CO}_{2}}^{b}=1.0 \mathrm{bar}$, in addition to the diffusivities and solubilities tabulated in Table I. We obtain $\mathrm{Ra}_{w} \approx 3 \times 10^{4}$, which is well above the critical value, $\mathrm{Ra}_{c}=1708$, for the onset of convection in an infinitely wide enclosure $\left(d / H_{w} \rightarrow \infty\right)$ [45,47]. The Schmidt number is large: $\mathrm{Sc}=v_{w} / D_{\mathrm{CO}_{2}}^{w}=568$. Therefore, our moderate- $\mathrm{Ra}_{w}$, high-Sc experiments should lie in the viscosity-dominated regime (also large-Prandtl or laminar regime [45], or regime $I_{u}$ in the Grossmann-Lohse theory [44]), where buoyancy forces are indeed balanced by viscous forces. We therefore expect a $\mathrm{Sh}_{w} \sim \mathrm{Ra}_{w}^{1 / 4}$ dependence [44,45], consistent with measurements of laminar Rayleigh-Bénard convection [48], and natural convection adjacent to horizontal surfaces [49,50], dissolving drops [22,23], or growing bubbles [24]. This laminar regime should not be mistaken for that of viscous convection of an infinite-Prandtl or Schmidt fluid in the limit of large Rayleigh numbers. For such a case, the scaling $\mathrm{Sh}_{w} \sim \mathrm{Ra}_{w}^{1 / 5}$ is expected between (no-slip) solid surfaces [51,52] (cf. regime $I_{\infty}^{>}$of the Grossmann-Lohse theory [53]) and $\mathrm{Sh}_{w} \sim \mathrm{Ra}_{w}^{1 / 3}$ between (zero shear stress) free surfaces $[51,52,54]$. However, the robustness of the $1 / 4$ exponent present in all the aforementioned cases of laminar convection hints that neither the no-shear boundary condition nor the cell geometry impact the scaling significantly. In our case, this is further justified by noting that the aspect ratio remains always close to unity, and the fact that bubble surfaces are prone to contamination by impurities naturally present in the ambient and can consequently behave as no slip [55]. Nevertheless, the prescribed scaling $\mathrm{Sh}_{w} \sim \mathrm{Ra}_{w}^{1 / 4}$ will be verified experimentally later in Sec. V.

Provided that variations in $\operatorname{Ra}_{w}(t)$ are sufficiently slow, we assume that the dependance of the quasisteady Sherwood number on the instantaneous Rayleigh number is of the form

$$
\mathrm{Sh}_{w}(t)=\left[1+k \frac{\mathrm{Ra}_{w}(t)}{\mathrm{Ra}_{c}}\right]^{1 / 4},
$$

where $\mathrm{Ra}_{c}=1708$ and $k=2.75 \pm 1.25$ is a dimensionless fitting coefficient that can be systematically obtained from the experimental measurements, as will be shown later in Sec. V. The fitting coefficient $k$ is expected to depend on Sc and the aspect ratio $d / H_{w}$ of the system but otherwise it is ideally a constant. Its variability across the experiments arises from several factors such as the different aspect ratios employed, uneven shapes of the bubble menisci, or the heterogeneous wetting state of the cylinder walls. In short, part of the variability is bound to the experimental uncertainty involving $V_{b}(t)$. Furthermore, the requirement that Eq. (4) should well capture the transition from pure diffusive transport $\left(\mathrm{Sh}_{w}=1\right.$ for $\left.\mathrm{Ra}_{w}<\mathrm{Ra}_{c}\right)$ to convection-dominated transport $\left(\mathrm{Sh}_{w} \sim \mathrm{Ra}_{w}^{1 / 4}\right.$ for $\mathrm{Ra}_{w} \gg \mathrm{Ra}_{c}$ ) is not at all important here, since all of our experiments correspond exclusively to the latter. To model details of the transition, an additional fitting parameter could be employed (cf. Ref. [22]).

At this point, we must invoke additional physical assumptions inspired by Howard's [56] and Malkus's [57] phenomenological treatment of turbulent Rayleigh-Bénard convection. First, we assume that most of the concentration drop $\Delta C_{\mathrm{CO}_{2}}^{w}$ occurs across the top diffusive boundary layer, whereas the convective region in the bulk of the water layer acts as a short circuit for mass transfer. The concentration drop across the bottom boundary layer is assumed to be negligible. This structure is sketched in Fig. 4(a). Second, we assume that the top boundary layer must always be in a "marginally stable state" once it develops. This means that the boundary layer cannot surpass (or shrink below) a critical or stable size $\delta\left(\mathrm{Ra}_{w}\right)$ which is such to ensure that the quasisteady mass transfer rate across the water layer is precisely given by Eq. (4). Note that the thickness of the 
(a)

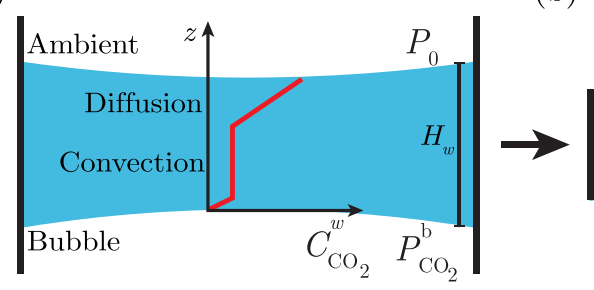

(b)

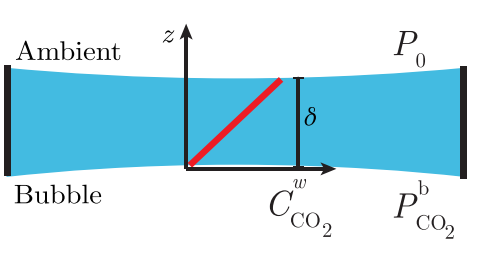

FIG. 4. (a) Sketch of the quasisteady concentration profile of $\mathrm{CO}_{2}$ across the water layer after the onset of convective dissolution. The main concentration drop occurs across the top diffusion boundary layer. Transport through the convective layer is assumed instant. The concentration drop across the bottom boundary layer is neglected. This is equivalent to (b), where the water layer is treated as a single diffusion layer of thickness $\delta(t) \sim H_{w} \operatorname{Ra}_{w}(t)^{-1 / 4}$, where $\mathrm{Ra}_{w}(t) \propto\left[P_{0}-P_{\mathrm{CO}_{2}}^{b}(t)\right]$, the difference in the partial pressure of $\mathrm{CO}_{2}$ in the $\mathrm{CO}_{2}-$ ambient and the bubble.

concentration boundary layer is connected to $\mathrm{Sh}_{w}$ by $\delta \sim H_{w} / \mathrm{Sh}_{w}$ [44,45]; hence, we see that $\delta / H_{w} \sim \mathrm{Ra}_{w}^{-1 / 4}$.

Under these circumstances, the quasisteady (fully developed) concentration profile across $\delta$ can be considered linear. The quasisteady flux is thus $J_{\mathrm{CO}_{2}}^{w b}=D_{\mathrm{CO}_{2}}^{w} \Delta C_{\mathrm{CO}_{2}}^{w} / \delta$. Inserting this result into the definition of $\mathrm{Sh}_{w}$ in Eq. (3), we determine the stable quasistationary thickness of the boundary layer to be

$$
\delta(t)=\frac{H_{w}}{\mathrm{Sh}_{w}(t)}=H_{w}\left[1+k \frac{\mathrm{Ra}_{w}(t)}{\mathrm{Ra}_{c}}\right]^{-1 / 4} .
$$

Therefore, we ultimately treat convection as the shortening of the effective diffusion layer from $H_{w}$ to $\delta(t)$, as illustrated in Fig. 4(b). This treatment could be applied in the modeling of the various different regimes in the $\mathrm{Sc}, \mathrm{Ra}_{w}$ parameter space [44] or even to situations where forced convection plays a role, in which case the governing relation in Eq. (4) should be adapted accordingly. In our numerical model, we simply solve the diffusion equation for $C_{\mathrm{CO}_{2}}^{w}$ over the domain $0<z<\delta(t)$, where $\delta(t)$ is reevaluated at every time step. The resulting numerical growth curves are plotted with white markers in Fig. 3(a) for cases (i) and (ii), with good general agreement. Equation (5) also explains why the bubble growth rate still decreases with $H_{w}$ despite the fact that $\mathrm{Ra}_{w} \propto H_{w}^{3}$. Indeed, it follows that $\delta \propto H_{w}^{1 / 4}$, and a longer diffusion distance translates into weaker fluxes and slower growth.

The good performance of the model extends to experiments at higher pressures, as shown in Fig. 5(a). Here, the growth rate of bubbles with postpressurization volumes $V_{f}=35 \mu \mathrm{L}$ under a water layer of $H_{w} \approx 4.5 \mathrm{~mm}$ are compared for pressure levels $P_{0}\left(t>t_{f}\right)=1.0,2.0$, and 3.1 bar. It becomes clear that $\dot{V}_{b}$ increases with pressure level $P_{0}$. This result, perhaps counterintuitive, is purely a consequence of the stronger convection, since $\mathrm{Ra}_{w} \propto\left(P_{0}-P_{\mathrm{CO}_{2}}^{b}\right) \sim P_{0}$. Note that in the absence of convection (pure diffusion), $\dot{V}_{b}$ remains fairly independent of $P_{0}$, since both the diffusive $\mathrm{CO}_{2}$ fluxes and the $\mathrm{CO}_{2}$ density in the bubble are proportional to $P_{0}$.

The validity of the quasisteady approximation of $\mathrm{Sh}_{w}(t)$ and $\delta(t)$ implied by the model in Eq. (5) can be assessed from the time evolution of $\delta(t)$ and $\mathrm{Ra}_{w}(t)$ for any particular experiment. We select the $P_{0}\left(t>t_{f}\right)=3.1$ bars experiment from Fig. 5(a) for this matter; the results are shown in Fig. 5(b). Before flushing and the onset of convection, $\delta / H_{w}=1$. At the start of the flushing stage, at $t=t_{f \text {-start }}$, the critical size for stability shrinks to $\delta / H_{w} \approx 0.22$ in accordance with Eq. (5), i.e., as established by the instantaneous $\operatorname{Ra}_{w}(t)$ at the flushing pressure of approximately 1 bar. Upon compression to $P_{0}=3.1$ bars shortly before $t_{f}, \delta(t)$ shrinks further accordingly. Thereafter, bubble growth and the concomitant increase in $P_{\mathrm{CO}_{2}}^{b}(t)$ causes $\delta(t)$ and $\mathrm{Ra}_{w}(t)$ to slowly increase and decay, respectively. The slow $\delta\left(t>t_{f}\right)$ dynamics supports the quasisteady approximation. 
(a)

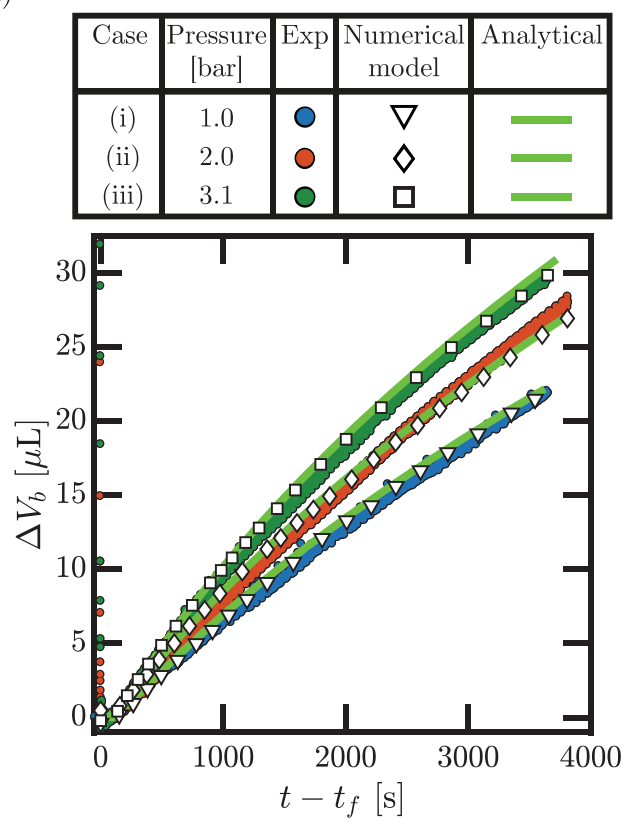

(b)

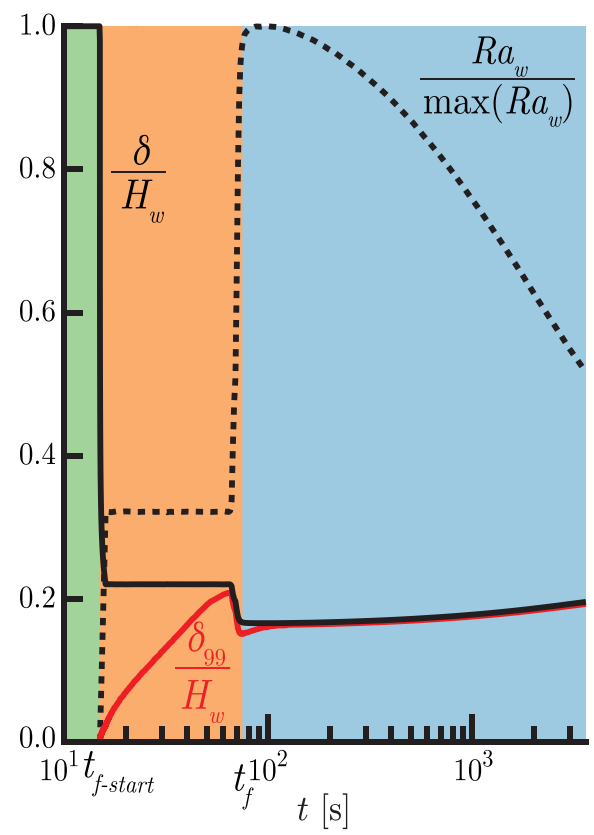

FIG. 5. (a) Effect of increasing the experiment pressure level $P_{0}\left(t>t_{f}\right)$ on the bubble growth dynamics. Water layer height is $H_{w} \approx 4.5 \mathrm{~mm}$ and postpressurization volumes are $V_{f} \approx 35 \mu \mathrm{L}$. Experimental, numerical, and analytical-Eq. (17)—results are compared. (b) Time evolution of the boundary layer thickness for marginal stability, $\delta(t)$ (black solid line), as established by its concomitant Rayleigh number (dotted line) according to the numerical model for experiment (iii) where $P_{0}\left(t>t_{f}\right)=3.1$ bars. The red solid line represents $\delta_{99}$, the distance over which $99 \%$ of the numerical concentration drop takes place. Equilibrium, flushing, and experiment stages are shaded accordingly (see Fig. 2).

The simplicity of this approach imposes no restriction to the initial diffusive development of the boundary layer in the model. We illustrate this in Fig. 5(b) by plotting the time evolution of $\delta_{99}$, the distance over which $99 \%$ of the concentration drop takes place. As soon as flushing begins at $t_{f-\text { start }}$, we assume that the ambient air in the tank is instantaneously replaced by $\mathrm{CO}_{2}$, which sets the top boundary condition to $C_{\mathrm{CO}_{2}}^{w}\left(H_{w}, t\right)=S_{\mathrm{CO}_{2}}^{w} P_{0}\left(t_{f-\text { start }}\right)$. The boundary layer evolves in a self-similar manner, and the concentration profiles can be well described by the self-similar solution

$$
C_{\mathrm{CO}_{2}}^{w}(z, t)=S_{\mathrm{CO}_{2}}^{w} P_{0}\left(t_{f-\text { start }}\right) \operatorname{erfc}\left(\frac{H_{w}-z}{\sqrt{4 D_{\mathrm{CO}_{2}}^{w}\left(t-t_{f-\text { start }}\right)}}\right) .
$$

The diffusive boundary layer $\delta_{99}(t)$ eventually attains the critical size $\delta$, after which $\delta_{99}=\delta$ and across it a linear concentration profile develops. Pressurization at $t_{f}$ would of course alter the selfsimilar development of the profiles in the strictest sense. Nonetheless, we see that the saturation time of $\delta_{99}$ is short-most of the $\delta_{99}$ development occurs within the short timeframe of the flushing stage itself. In fact, this saturation time, namely, the time required for the self-similar regime to transition towards the $\delta$-bounded, quasisteady regime, can be estimated as $0.3 \delta_{f}^{2} / D_{\mathrm{CO}_{2}}^{w}$, where $\delta_{f} \equiv$ $\delta\left(t_{f}\right)$ is the effective diffusion length (see Appendix A, Fig. 10). The concentration profiles for a previous experiment [where $P_{0}\left(t>t_{f}\right)=1$ bar] drawn in Fig. 3(b) clearly demonstrate the rapid initial development of the boundary layer in convective experiments. On the other hand, for diffusive experiments [see profiles in Fig. 3(b)], the boundary layer saturation time is $0.3 H_{w}^{2} / D_{\mathrm{CO}_{2}}^{w}$, i.e., an order of magnitude larger, which results in a noticeably longer initial transient period of slow bubble growth. 
Finally, we turn our attention to the gas composition of the bubbles over time [see Fig. 3(c)]. The air $\left(\mathrm{O}_{2}\right.$ and $\mathrm{N}_{2}$ gas) content in the growing bubbles appears to remain virtually unchanged throughout the duration of the experiments by virtue of the low solubility of air in water. This observation is especially true for the bubbles growing at $P_{0}\left(t>t_{f}\right)=1$ bar-Fig. 3(c) preciselysince the concomitant air fluxes are weakest then. Therefore, to a good approximation, the growth in bubble volume can be fully attributed to the uptake of $\mathrm{CO}_{2}$ from the water layer. This result is pivotal in the derivation of the analytical solutions for bubble growth presented in the next section.

\section{B. Analytical solutions for bubble growth}

In the analysis that follows, we consider the bubble volume dynamics for $t>t_{f}$, i.e., after flushing and pressurization. The initial bubble volume is thus $V_{b}\left(t_{f}\right) \equiv V_{f}$. We denote the experiment pressure level $P_{0}\left(t>t_{f}\right)$ simply as $P_{0}$, which is treated as constant in all regards. It follows that the partial pressure of $\mathrm{CO}_{2}$ in the ambient is $P_{\mathrm{CO}_{2}}^{0}\left(t>t_{f}\right)=P_{0}$ precisely. Furthermore, we assume that the flushing and pressurization stage takes place instantaneously at $t_{f}$, meaning that at $t<t_{f}$ we consider equilibrium conditions everywhere in the system. This is reasonable since the flushing period $\left(t_{f}-t_{f-s t a r t}\right)$ typically lasts $60 \mathrm{~s}$; i.e., it is quite short in comparison to the relevant characteristic diffusion times.

Finally, we treat air as insoluble; i.e., we neglect the flux of $\mathrm{O}_{2}$ or $\mathrm{N}_{2}$ across the bubble or water layer. The number of moles of gas in the bubble can be then decomposed as

$$
n_{b}(t)=n_{\mathrm{CO}_{2}}^{b}(t)+n_{\mathrm{N}_{2}}^{b}\left(t_{f}\right)+n_{\mathrm{O}_{2}}^{b}\left(t_{f}\right),
$$

with, initially, $n_{\mathrm{CO}_{2}}^{b}\left(t_{f}\right)=0$. We can then transform the above equation into a pressure-volume relationship. To do so, we make use of the ideal gas law, $P_{0} V_{b}=n_{b} R T_{0}$, and the definition of the partial pressure of $\mathrm{CO}_{2}$ in the bubble, $P_{\mathrm{CO}_{2}}^{b}=n_{\mathrm{CO}_{2}}^{b} P_{0} / n_{b}$. Noting that $P_{0} V_{f}=n_{b}\left(t_{f}\right) R T_{0}$ initially, we obtain the following relationship:

$$
1-\frac{P_{\mathrm{CO}_{2}}^{b}(t)}{P_{0}}=\frac{V_{f}}{V_{b}(t)} .
$$

Next, recalling the definition of $\mathrm{Sh}_{w}$ [Eq. (3)], we find it useful to nondimensionalize the net molar flux into the bubble, $\dot{n}_{b} /\left(\pi d^{2} / 4\right)$, in the same manner. We refer to it as the dimensionless bubble growth rate:

$$
\phi_{b}(t) \equiv \frac{\dot{n}_{b}(t)}{\pi d^{2} / 4} \frac{H_{w}}{D_{\mathrm{CO}_{2}}^{w} \Delta C_{\mathrm{CO}_{2}}^{w}(t)} .
$$

Substituting in the ideal gas law $\dot{n}_{b}=P_{0} \dot{V}_{b} / R T_{0}$, using Henry's law $\Delta C_{\mathrm{CO}_{2}}^{w}(t)=S_{\mathrm{CO}_{2}}^{w}\left[P_{0}-P_{\mathrm{CO}_{2}}^{b}(t)\right]$ and relationship (8), Eq. (9) becomes

$$
\phi_{b}(t)=\frac{\dot{V}_{b}(t)}{Q} \frac{V_{b}(t)}{V_{f}}, \quad \text { where } \quad Q \equiv D_{\mathrm{CO}_{2}}^{w} \frac{\pi d^{2}}{4 H_{w}}\left(S_{\mathrm{CO}_{2}}^{w} R T_{0}\right) .
$$

The dimensional quantity $Q$ can be regarded as a characteristic volume flow rate and it is a constant. Finally, we introduce the dimensionless time coordinate, namely, in multiples of the diffusion time across the water layer,

$$
\tau \equiv \frac{t-t_{f}}{H_{w}^{2} / D_{\mathrm{CO}_{2}}^{w}},
$$

which will mainly serve as an argument to the transient flux functions (see Appendix A). The transient flux functions will be shown to be fundamental in the description of the initial transient growth of the bubble. 


\section{Diffusive growth}

A fully developed (linear) concentration profile across the full height water layer $H_{w}$ implies, by definition, a quasisteady $\mathrm{Sh}_{w}=1$. However, there is an initial period of boundary layer development (lasting $\sim H_{w}^{2} / 4 D_{\mathrm{CO}_{2}}^{w}$ ), during which $\mathrm{Sh}_{w}(t)$ evolves from zero to 1 . We can formally capture the transient rate of mass transfer through the inclusion of a transient correction term $f^{\prime}(\tau)$ (see Appendix A). It derives from the analytical computation the diffusive flux across a layer of finite height with initial uniform zero concentration, and assuming one end of the layer (ambient interface) is at a constant concentration while the remaining end (bubble interface) remains at zero concentration. The latter assumption that $P_{\mathrm{CO}_{2}}^{b}(t)$ remains zero is completely justified given that the fractional bubble growth is indeed small during the transient period. In such a case,

$$
\mathrm{Sh}_{w}=1-f^{\prime}(\tau)
$$

where $f^{\prime}(\tau)$ is given in Eq. (A2). $\mathrm{Sh}_{w}(\tau)$ is plotted in Fig. 10(a). Mass conservation implies that $\phi_{b}=\mathrm{Sh}_{w}$. Equating Eqs. (10) and (12) results in an integrable differential equation for $V_{b}(t)$, subject to the initial condition $V_{b}\left(t_{f}\right)=V_{f}$. Integration yields

$$
V_{b}(t)=V_{f} \sqrt{1+\frac{2 Q}{V_{f}}\left[\left(t-t_{f}\right)-\frac{H_{w}^{2}}{D_{\mathrm{CO}_{2}}^{w}} f(\tau)\right]},
$$

where the transient flux integral function $f(\tau)$ is defined in Eq. (A3) and has limits $f(0)=0$ and $f(\infty)=1 / 6$. As expected, $V_{b}$ does not depend on the ambient pressure $P_{0}$. The analytical solution (13) is plotted in Fig. 3(a) with good agreement with its experimental and numerical counterparts, (iii) and (iv).

\section{Convective growth}

The $\mathrm{Sh}_{w}\left(\mathrm{Ra}_{w}\right)$ dependence proposed in Eq. (4) can be simplified to $\mathrm{Sh}_{w}=\left(k \mathrm{Ra}_{w} / \mathrm{Ra}_{c}\right)^{1 / 4}$ since

$$
\operatorname{Ra}_{w}(t)=\frac{\lambda{ }_{\mathrm{CO}_{2}}^{w} S_{\mathrm{CO}_{2}}^{w} g}{v_{w} D_{\mathrm{CO}_{2}}^{w}} H_{w}^{3}\left[P_{0}-P_{\mathrm{CO}_{2}}^{b}(t)\right]
$$

is indeed large. We define $\mathrm{Ra}_{f} \equiv \mathrm{Ra}_{w}\left(t_{f}\right)$ as the initial (and maximum) $\mathrm{Ra}_{w}$ under the assumption that at $t_{f}$ there is still no $\mathrm{CO}_{2}$ in the bubble, $P_{\mathrm{CO}_{2}}^{b}\left(t_{f}\right)=0$. Thereafter, $\operatorname{Ra}_{w}(t)$ has been shown to decay with bubble growth [cf. Fig. 5(b)] due to the concomitant increase in $P_{\mathrm{CO}_{2}}^{b}(t)$. Combining the relationship in Eq. (8) with Eq. (14) gives $\mathrm{Ra}_{w}(t) / \mathrm{Ra}_{f}=V_{f} / V_{b}(t)$. Hence, we can relate the quasisteady Sherwood number to the bubble volume by

$$
\mathrm{Sh}_{w}(t)=\beta\left(\frac{V_{f}}{V_{b}(t)}\right)^{1 / 4}, \quad \text { with } \quad \beta \equiv\left(\frac{k \mathrm{Ra}_{f}}{\mathrm{Ra}_{c}}\right)^{1 / 4} .
$$

The dimensionless parameter $\beta$ can be regarded as the quasisteady Sherwood number that could be potentially attained at $t_{f}$ upon the immediate onset of quasisteady convection (infinitely short transient period). Therefore, recalling that $\delta=H_{w} / \mathrm{Sh}_{w}$, the initial critical size of the boundary layer is set by $\delta_{f}=H_{w} / \beta$.

The initial transient regime before the onset of quasisteady convection can be accounted for with an identical correction term as previously done in the diffusive case, except that we now consider the boundary layer development over the length $\delta_{f}$ instead of $H_{w}$. Accordingly, the argument of the transient functions $f^{\prime}$ in Eq. (A2) and $f$ in Eq. (A3) is now equal to $\beta^{2} \tau$. The transient-corrected Sherwood number becomes

$$
\mathrm{Sh}_{w}(t)=\beta\left[1-f^{\prime}\left(\beta^{2} \tau\right)\right]\left(\frac{V_{f}}{V_{b}(t)}\right)^{1 / 4}
$$


Mass conservation implies $\phi_{b}=\mathrm{Sh}_{w}$. Equating Eqs. (10) and (16) followed by integration from $V_{b}\left(t_{f}\right)=V_{f}$ results in

$$
V_{b}(t)=V_{f}\left[1+\frac{9 Q}{4 V_{f}} \beta\left\{\left(t-t_{f}\right)-\frac{H_{w}^{2} / D_{\mathrm{CO}_{2}}^{w}}{\beta^{2}} f\left(\beta^{2} \tau\right)\right\}\right]^{4 / 9} .
$$

The analytical solution is compared with experiments and numerical solutions in Fig. 3(a) [curves (i) and (ii)] and Fig. 5(a), displaying a very decent performance in all cases. It should be pointed out that the analytical solution intentionally shares the same fitting parameter $k$ (i.e., through $\beta$ ) as its numerical counterpart, also bearing in mind that $k$ may vary from one experiment to another within the aforementioned bounds.

\section{CONVECTIVE MASS TRANSFER: SCALING}

Our theoretical framework for convective dissolution is strongly based on Eq. (4) in the limit of Eq. (15), namely, on the assumption that the quasisteady Sherwood number depends on the instantaneous Rayleigh number as $\mathrm{Sh}_{w} \sim \mathrm{Ra}_{w}^{1 / 4}$. The validity of this assumption can be verified experimentally without the need for a supporting numerical model. This requires the approximation of regarding air as insoluble. In addition, we abbreviate the pressure level $P_{0}\left(t>t_{f}\right)$ as $P_{0}$, which is assumed to be constant. From earlier analyses (Sec. IV B), it follows that the instantaneous Rayleigh number

$$
\operatorname{Ra}_{w}(t)=\operatorname{Ra}_{f} \frac{V_{f}}{V_{b}(t)}, \quad \text { where } \quad \operatorname{Ra}_{f} \equiv \frac{\lambda_{\mathrm{CO}_{2}}^{w} S_{\mathrm{CO}_{2}}^{w} g}{v_{w} D_{\mathrm{CO}_{2}}^{w}} H_{w}^{3} P_{0},
$$

and the dimensionless bubble growth rate defined in Eqs. (9) and (10) (repeated here for convenience),

$$
\phi_{b}(t)=\frac{\dot{V}_{b}(t)}{Q} \frac{V_{b}(t)}{V_{f}}, \quad \text { where } \quad Q \equiv D_{\mathrm{CO}_{2}}^{w} \frac{\pi d^{2}}{4 H_{w}}\left(S_{\mathrm{CO}_{2}}^{w} R T_{0}\right),
$$

can both be evaluated purely from the experimental data for $t>t_{f}$.

The result is shown in Fig. 6, where the time-averaged $\phi_{b}$ is plotted against the time-averaged $\mathrm{Ra}_{w}$ for every single experiment. Each colored marker represents an individual binary experiment; the grey markers belong to the ternary (water-bubble-alkane) experiments and are not expected to follow Eq. (4) as will be discussed later. The time averages are computed for $t-t_{f}>1100 \mathrm{~s}$ to discard the initial transient growth of the bubble. The error bars indicate the range over which $\phi_{b}(t)$ and $\mathrm{Ra}_{w}(t)$ decay during that particular experiment [cf. Fig. 5(b)]. In addition, the $\mathrm{Sh}_{w}\left(\mathrm{Ra}_{w}\right)$ dependence proposed in Eq. (4) is represented by the green solid curve, alongside its asymptotic slope of $1 / 4$. The shaded region around the curve delimits the range of the fitting parameter, $k=$ $2.75 \pm 1.25$, previously considered.

Figure 6(a) shows that the binary experiments fall in the asymptotic segment of the shaded $\mathrm{Sh}_{w}\left(\mathrm{Ra}_{w}\right)$ region. This results from mass conservation, which for the binary experiments can be expressed as $\left\langle\phi_{b}\right\rangle=\mathrm{Sh}_{w}$, indicating that the $\mathrm{CO}_{2}$ flux across the water layer must be equal to the $\mathrm{CO}_{2}$ accumulation rate in the bubble. The asymptotic segment of the shaded region is described by $\mathrm{Sh}_{w}=a \mathrm{Ra}_{w}^{1 / 4}$, where $a=0.2 \pm 0.03$. Interestingly, our prefactor $a \approx 0.2$ seems to coincide with that obtained by Malkus [48] in one of the first laminar Rayleigh-Bénard convection experiments ever conducted. It is also in complete agreement with the $0.33 \mathrm{Sc}^{-1 / 12} \approx 0.19$ prefactor of the $I_{u}$ regime of the Grossmann-Lohse theory of Rayleigh-Bénard convection in a cylindrical cell [44]. Recent experiments of $\mathrm{CO}_{2}$ bubbles growing in carbonated water [24] suggest comparable values ( $a \approx 0.3$, with the bubble radius as the relevant length scale). Dissolving sessile alcohol droplets in water [22] yield a somewhat higher exponent $(a \approx 0.6)$. On the other hand, natural convection measurements over horizontal plates, at a quite different geometry, at $\operatorname{Pr} \sim 1$ result in prefactors (based on the plate diameter) approximately fourfold larger than ours [49,50]. 
(a)

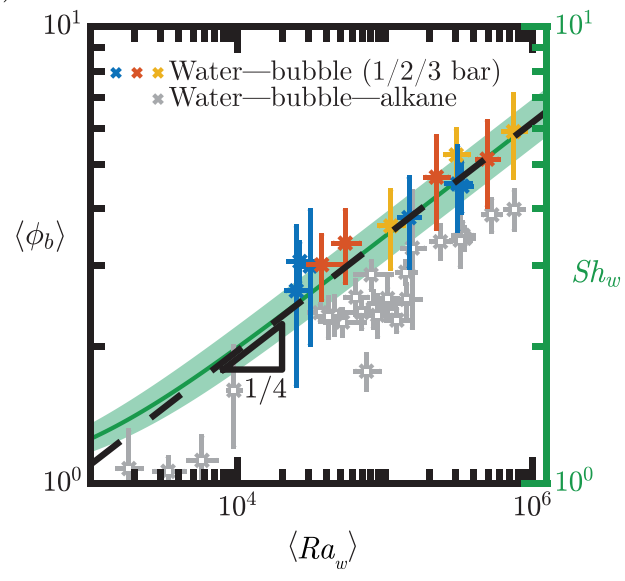

(b)

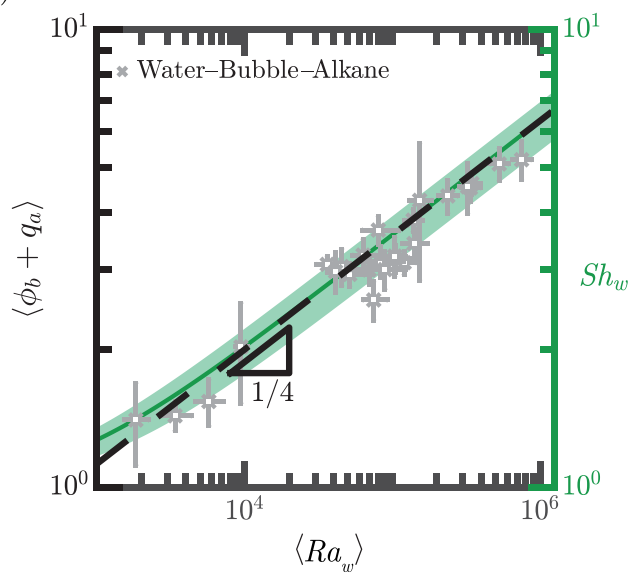

FIG. 6. (a) Time-averaged dimensionless bubble growth rate as a function of the time-averaged $\mathrm{Ra}_{w}$ for every single water-bubble experiment (colored markers) and water-bubble-alkane experiment (grey markers). The error bars indicate the range over which $\phi_{b}(t)$ and $\operatorname{Ra}_{w}(t)$ decay during that particular experiment. The solid green line and surrounding shaded region provide the $\mathrm{Sh}_{w}\left(\mathrm{Ra}_{w}\right)$ dependence in Eq. (4): $\mathrm{Sh}_{w}=(1+$ $\left.k \mathrm{Ra}_{w} / \mathrm{Ra}_{c}\right)^{1 / 4}$, with $k=2.75 \pm 1.25$. (b) Bubble growth rate of the water-bubble-alkane experiments corrected with $q_{a}$, the dimensionless $\mathrm{CO}_{2}$ leakage flux from the bubble to the alkane according to Eq. (25).

\section{WATER-BUBBLE-ALKANE SYSTEM: BUFFERING EFFECT}

Next, we turn our attention to the fact that all the ternary experiments fall systematically below the $\mathrm{Sh}_{w}\left(\mathrm{Ra}_{w}\right)$ curve in Fig. 6(a). Indeed, $\left\langle\phi_{b}\right\rangle<\left\langle\mathrm{Sh}_{w}\right\rangle$ is a consequence of the onset of a $\mathrm{CO}_{2}$ leakage flux from the bubble into the alkane layer below. Mass conservation now reads $\mathrm{Sh}_{w}=$ $\phi_{b}+q_{a}$, where the (dimensionless) leakage flux $q_{a}(t)$ can be inferred purely from the experimental data under a set of simplifying assumptions. The result is shown in Fig. 6(b). Upon correcting $\left\langle\phi_{b}\right\rangle$ with $\left\langle\phi_{b}+q_{a}\right\rangle$, the experimental points are coherently shifted up into the $\operatorname{Sh}_{w}\left(\mathrm{Ra}_{w}\right)$ region.

The expression for $q_{a}(t)$ is provided in Eq. (25) below. It will be derived in this section, which explores the stabilizing or "buffering" effect of the alkane layer on bubble growth in greater detail.

The dissolution of $\mathrm{CO}_{2}$ into hexadecane is known to induce density gradients which similarly result in its enhanced transport [58]. Indeed, density measurements of hexadecane $+\mathrm{CO}_{2}$ mixtures $\left(\rho_{a}\right)[59]$ and water $+\mathrm{CO}_{2}\left(\rho_{w}\right)$ mixtures $[43,60]$ at the same high pressure range $\left(P_{0}>10 \mathrm{MPa}\right)$ and room temperature conditions coincidentally yield a solutal expansion coefficient

$$
\lambda_{\mathrm{CO}_{2}}^{a / w} \equiv\left(\frac{1}{\rho_{a / w}} \frac{\partial \rho_{a / w}}{\partial C_{\mathrm{CO}_{2}}^{a / w}}\right)_{P_{0}, T_{0}}
$$

of approximately $10 \mathrm{~cm}^{3} / \mathrm{mol}$ in both cases. Note that superscript or subscript $a$ is used to refer to the alkane ( $n$-hexadecane) layer or medium, in the same manner as $w$ refers to water. Since $\lambda_{\mathrm{CO}_{2}}^{w}$ is known to depend very weakly on $P_{0}$ [43] (verified by the fact that Ref. [61] reports $\lambda_{\mathrm{CO}_{2}}^{w}=$ $10.9 \mathrm{~cm}^{3} /$ mol under atmospheric $P_{0}$ ), it is then reasonable to assume that $\lambda_{\mathrm{CO}_{2}}^{a} \approx \lambda_{\mathrm{CO}_{2}}^{w}$ also holds true in our low- $P_{0}$ conditions.

To determine if convective dissolution across the hexadecane layer constitutes a dominant effect, we must estimate the characteristic Rayleigh number across it, $\mathrm{Ra}_{a}$. At the bubble-alkane interface, the surface concentration is $S_{\mathrm{CO}_{2}}^{a} P_{\mathrm{CO}_{2}}^{b}(t)$. At the bottom of the layer a no-flux boundary condition holds. Assuming pure diffusive transport, and provided that the increase in $P_{\mathrm{CO}_{2}}^{b}(t)$ is sufficiently slow, the maximum (or characteristic) concentration difference across the alkane layer corresponds to that at the time $t^{*}$ where self-similarity is broken therein, i.e., when the $\mathrm{CO}_{2}$ concentration at the 
(a)

\begin{tabular}{|c|c|c|c|c|c|}
\hline Case & System & $H_{w}[\mathrm{~mm}]$ & Exp & $\begin{array}{c}\text { Numerical } \\
\text { model }\end{array}$ & $\begin{array}{c}\text { Analytical } \\
\text { /ODE }\end{array}$ \\
\hline (i) & Binary & 3.0 & $\bullet$ & $\nabla$ & \\
(ii) & & 6.5 & $\bigcirc$ & $\diamond$ & \\
(iii) & Ternary & 3.4 & $\bullet$ & $\boldsymbol{\nabla}$ & \\
(iv) & & 6.5 & 0 & $\diamond$ & \\
\hline
\end{tabular}

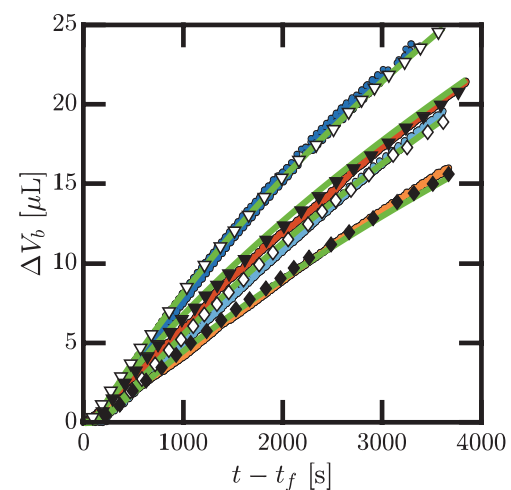

(b)

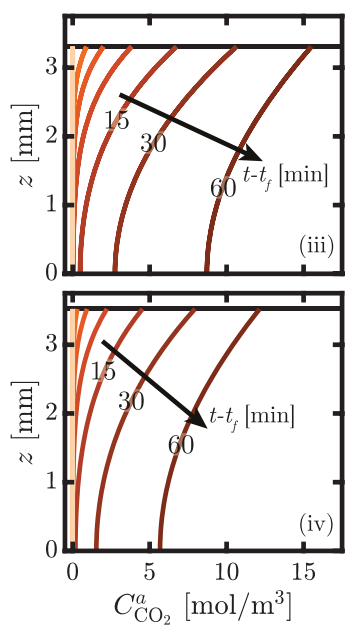

FIG. 7. (a) Comparison of the bubble growth dynamics for water-bubble system [cases (i) and (ii)] and ternary water-bubble-alkane systems [cases (ii) and (iv)] for two distinct water layer heights. The alkane layer height is $H_{a} \approx 3.5 \mathrm{~mm}$. In all four cases, $V_{f}=35 \mu \mathrm{L}$ and $P_{0}=1$ bar. Experiments (i) and (ii) are overlaid with numerical and analytical (17) solutions, experiments (iii) and (iv) with numerical and ordinary differential equation (ODE) (27) solutions. (b) Time evolution of the $\mathrm{CO}_{2}$ concentration profile in the alkane layer according to the numerical model for case (iii) (top panel) and case (iv) (bottom panel).

bottom of the alkane layer starts to rise. $\mathrm{CO}_{2}$ accumulates in the alkane layer thereon by virtue of the no-flux boundary condition. The time $t^{*}$ is determined by $t^{*}-t_{f} \approx 0.3 H_{a}^{2} / D_{\mathrm{CO}_{2}}^{a}$ (see Appendix A). Furthermore, we approximate air as insoluble in hexadecane, whereby $P_{b}=P_{0} \Delta V_{b} / V_{b}$ from Eq. (8) applies.

The resulting expression for $\mathrm{Ra}_{a}$ can be insightfully deployed in relation to the maximum Rayleigh number across the water layer, $\mathrm{Ra}_{f}$ [see Eq. (18)]. Neglecting the small differences in the solubilities, diffusivities, and solutal expansion coefficients of $\mathrm{CO}_{2}$ in water and in hexadecane (see Table I), we can write

$$
\frac{\mathrm{Ra}_{a}}{\operatorname{Ra}_{f}} \approx \frac{\Delta V_{b}}{V_{b}} \frac{H_{a} v_{w}}{H_{w} v_{a}} .
$$

For a typical experiment with $V_{f}=35 \mu \mathrm{L}$ and $H_{a}=3 \mathrm{~mm}$, we find $\left(t^{*}-t_{f}\right) \sim 1200 \mathrm{~s}$ and, from earlier figures, $\Delta V_{b}\left(t^{*}\right) \approx 5 \mu \mathrm{L}$, hence $\Delta V_{b}\left(t^{*}\right) / V_{b}\left(t^{*}\right) \approx 1 / 8$. Taking $H_{w} \approx H_{a}$, and noting that $v_{w} / v_{a} \approx 1 / 4$, we finally obtain a characteristic magnitude of $\mathrm{Ra}_{a} / \mathrm{Ra}_{f} \sim 3 \%$. This always translates to $\mathrm{Ra}_{a}<10^{4}$, i.e., $\mathrm{Ra}_{a} \sim \mathrm{Ra}_{c}$ at most. Therefore, under the current experimental conditions, diffusion can be safely assumed to remain the dominant transport mechanism across the alkane layer. The numerical model can then be straightforwardly extended to incorporate the presence of the alkane layer (see Appendix B), by solving the one-dimensional diffusion equation for all three species $\left(j=\mathrm{CO}_{2}, \mathrm{O}_{2}\right.$, and $\left.\mathrm{N}_{2}\right)$ over the whole height of the alkane layer. The resulting interfacial fluxes from the bubble to the alkane, $J_{j}^{b a}$, modify mass conservation to $J_{j}^{w b}-J_{j}^{b a}=4 \dot{n}_{j}^{b} / \pi d^{2}$ [cf. the discussion above Eq. (2)].

Figure 7(a) compares the bubble growth dynamics of two binary experiments at $P_{0}\left(t>t_{f}\right)=$ 1 bar [same cases (i) and (ii) from Fig. 3] with their equivalent ternary systems with $H_{a} \approx 3.5 \mathrm{~mm}$ under otherwise the same conditions. The buffering effect of the $n$-hexadecane layer on bubble growth first introduced in Fig. 2 is clearly observed again. The good performance of the numerical solution validates, once more, the assumption of negligible convective dissolution across the alkane 
layer. On another note, the transient nature of $J_{j}^{b a}(t)$ can be inferred from the time evolution of the numerical $\mathrm{CO}_{2}$ concentration profiles in the alkane layer shown in Fig. 7(b). Quasisteady or self-similar profiles or fluxes are never attained. This is a consequence of the unsteady interfacial concentration $C_{\mathrm{CO}_{2}}^{a}\left(H_{a}, t\right)$ being strictly an increasing function of time and the no-flux boundary condition on $z=0$. Notice that $\mathrm{CO}_{2}$ starts accumulating in the alkane layer after a time of approximately $15 \mathrm{~min}$, in accordance with our previous estimation.

The added analytical complexity brought by the transient nature of the $\mathrm{CO}_{2}$ transport across the alkane layer can be witnessed, for example, in the unmanageable time-integral-laden mathematical expression of the concentration profile across a finite sheet subject to a no-flux boundary condition and variable surface concentration [62]. However, noting that on the time scale of our experiments the hexadecane layer hardly becomes saturated [cf. Fig. 7(b)], we may choose to ignore the effect of the no-flux boundary. We do so by treating the hexadecane layer as a semi-infinite medium. Not only is this a reasonable simplification, it also poses a problem of particular interest. In such a case, and treating air as insoluble, we can reduce the model formulation to a single ordinary differential equation for $V_{b}(t)$ as will be shown in the next section.

\section{A. Dynamic equation for bubble growth}

Approximating air as insoluble renders Eq. (8) which allows us to relate the surface concentration of $\mathrm{CO}_{2}$ in the alkane layer at the alkane-bubble interface to the instantaneous bubble volume by

$$
C_{s}(t)=S_{\mathrm{CO}_{2}}^{a} P_{\mathrm{CO}_{2}}^{b}(t)=S_{\mathrm{CO}_{2}}^{a} P_{0}\left[1-\frac{V_{f}}{V_{b}(t)}\right],
$$

where $P_{0}$ here abbreviates the constant pressure level $P_{0}\left(t>t_{f}\right)$. It should be noted that, unlike in water, the solubilities of air and $\mathrm{CO}_{2}$ in $n$-hexadecane are quite comparable (see Table I). Therefore, the approximation of neglecting the flux of air into the hexadecane layer is mostly valid for $P_{0}=$ 1 bar because the hexadecane layer is initially saturated with air at that pressure. Presurrization beyond this value results in the immediate undersaturation of the latter; raising $P_{0}$ further eventually yields fluxes of air and $\mathrm{CO}_{2}$ of similar magnitude.

Next, treating the alkane layer as semi-infinite fully removes the length scale $H_{a}$ from the problem, which allows us to conveniently work with the water-layer time $\tau$ defined in Eq. (11) instead. The $\mathrm{CO}_{2}$ diffusion flux from the bubble to the semi-infinite alkane layer with a time-dependent surface concentration is given by (see, e.g., Ref. [63])

$$
J_{\mathrm{CO}_{2}}^{b a}=\frac{D_{\mathrm{CO}_{2}}^{a}}{H_{w}}\left[\frac{1}{\sqrt{\pi}} \int_{0}^{\tau} \frac{1}{\sqrt{\tau-u}} \frac{\mathrm{d} C_{s}}{\mathrm{~d} u} \mathrm{~d} u\right] .
$$

Identical history integral flux terms to the one above can be found deployed in previous analytical treatments concerning the growth and dissolution of drops and/or bubbles with unsteady surface concentrations [64-65]. The integral describes the so-called history effect, i.e., the contribution of past mass transfer events on the current growth dynamics. The fact that the recent history contributes the most, $1 / \sqrt{\tau-u} \rightarrow \infty$ as $u \rightarrow \tau$, can be used to evaluate the time integral in our case. Since the bubble growth dynamics are quite steady (no abrupt or fast changes in $\dot{V}_{b}$ ), we can approximate $\mathrm{d} C_{s} / \mathrm{d} u$ as the current time derivative $\mathrm{d} C_{s}(\tau) / \mathrm{d} \tau$ irrespective of $u$. Analytical integration is then possible, and the flux becomes

$$
J_{\mathrm{CO}_{2}}^{b a}=\frac{D_{\mathrm{CO}_{2}}^{a}}{H_{w}}\left[2 \sqrt{\frac{\tau}{\pi}} \frac{\mathrm{d} C_{s}}{\mathrm{~d} \tau}\right]=\left(\frac{2 D_{\mathrm{CO}_{2}}^{a} S_{\mathrm{CO}_{2}}^{a} P_{0} V_{f}}{\sqrt{\pi D_{\mathrm{CO}_{2}}^{w}}}\right) \frac{\dot{V}_{b}(t)}{V_{b}(t)^{2}} \sqrt{t-t_{f}},
$$

where use of Eq. (11) and Eq. (22) has been made. The dimensionless flux, $q_{a}$, into the alkane (normalized by the water-layer diffusive flux, in coherence with $\mathrm{Sh}_{w}$ and $\phi_{b}$ ) is

$$
q_{a}(t) \equiv \frac{J_{\mathrm{CO}_{2}}^{b a}(t) H_{w}}{D_{\mathrm{CO}_{2}}^{w} \Delta C_{\mathrm{CO}_{2}}^{w}(t)}=\sqrt{T^{a w}} \sqrt{t-t_{f}} \frac{\dot{V}_{b}}{V_{b}}, \quad \text { with } \quad \sqrt{T^{a w}}=\frac{2 H_{w}}{\sqrt{\pi D_{\mathrm{CO}_{2}}^{w}}} \frac{D_{\mathrm{CO}_{2}}^{a}}{D_{\mathrm{CO}_{2}}^{w}} \frac{S_{\mathrm{CO}_{2}}^{a}}{S_{\mathrm{CO}_{2}}^{w}}
$$


(a)

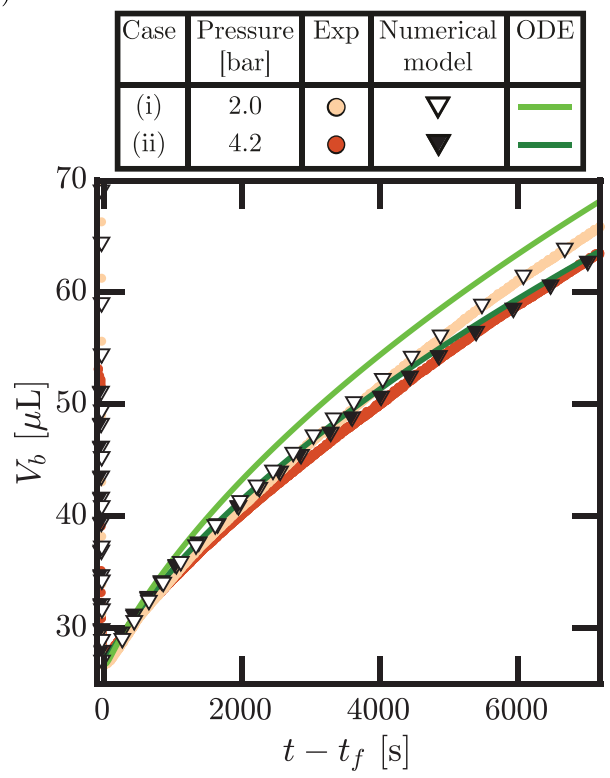

(b)

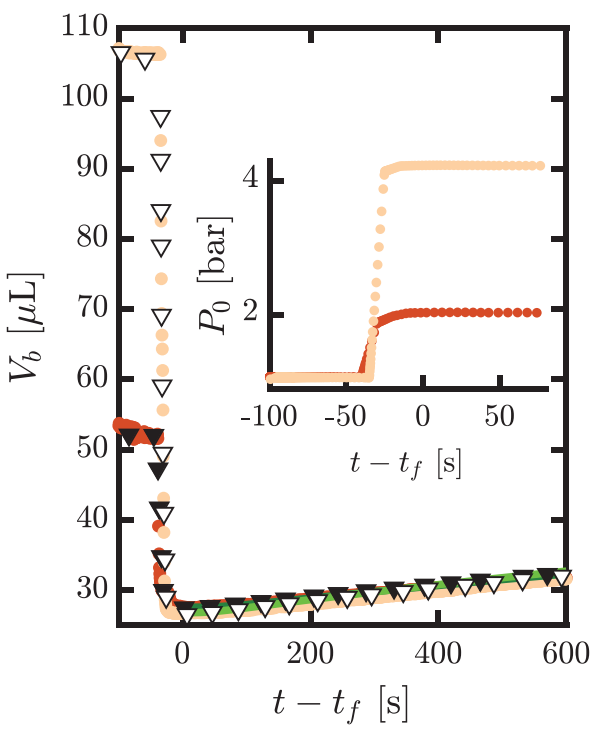

FIG. 8. (a) Bubble growth dynamics for the water-bubble-alkane system at higher pressure levels $\left(V_{f}=\right.$ $27 \mu \mathrm{L}$ ). Case (i), $P_{0}=2.0$ bars, $H_{w}=3.0 \mathrm{~mm}, H_{a}=3.2 \mathrm{~mm}$; case (ii), $P_{0}=4.2$ bars, $H_{w}=3.0 \mathrm{~mm}, H_{a}=$ $3.2 \mathrm{~mm}$. Experimental, numerical, and ODE (27) results are compared. (b) Close-up of the pressurization stage and initial growth. Inset: Concomitant pressure time history.

Here $T^{a w}$ is a time constant which depends purely on the system properties. The remaining ingredients have been derived in Sec. IV B; these are naturally $\phi_{b}$ and $\mathrm{Sh}_{w}$, whose expressions are given in Eq. (9) and Eq. (16), respectively. Finally, mass conservation yields

$$
\phi_{b}=\mathrm{Sh}_{w}-q_{a} .
$$

Substituting the expressions in Eqs. (9), (16), and (25), the mass balance becomes

$$
\frac{\dot{V}_{b}}{Q} \frac{V_{b}}{V_{f}}=\left\{\beta\left[1-f^{\prime}\left(\beta^{2} \tau\right)\right]\left(\frac{V_{f}}{V_{b}}\right)^{1 / 4}\right\}-\left\{\sqrt{T^{a w}} \sqrt{t-t_{f}} \frac{\dot{V}_{b}}{V_{b}}\right\},
$$

which constitutes an ordinary nonlinear differential equation for $V_{b}\left(t>t_{f}\right)$ that can be numerically integrated subject to the initial condition $V_{b}\left(t_{f}\right)=V_{f}$. The solution to Eq. (27) is plotted in Fig. 7(a) for cases (iii) and (iv), where the ODE solution shares the same fitting coefficient $k$ as its numerical counterpart. Overall, excellent agreement is observed, despite the underlying simplifications. We also point out that the flux $q_{a}(t)$ in Eq. (25) is independent of $k$ (or any other fitting parameter) and it can be evaluated directly from the experimental measurements, as done for Fig. 6(b).

Figure 8(a) shows the bubble growth dynamics of the water-bubble-alkane system $\left(H_{w} \approx H_{a} \approx\right.$ $3 \mathrm{~mm}$ ) at higher experiment pressure levels, namely, at $P_{0}=2.0$ and 4.2 bars. Interestingly, the alkane buffering effect also visibly diminishes the dependence of the growth rate $V_{b}$ on $P_{0}$. The separation of the growth curves is indeed less pronounced than in the binary system [see Fig. 5(a)]. The limitation of the ODE solution becomes apparent at these higher pressures. The ODE overestimates the growth rate, a consequence of neglecting the dissolution flux of air from the bubble into the alkane. The deviation worsens with increasing $P_{0}$, i.e., with the air flux magnitude. On another note, the accuracy of the numerical model in reproducing the initial pressure-bound dynamics is highlighted in Fig. 8(b), which shows a close-up of the initial minutes of bubble growth and the preceding pressurization stage. Bubble dissolution for $t>t_{f}$ is not observed, despite the fact that 


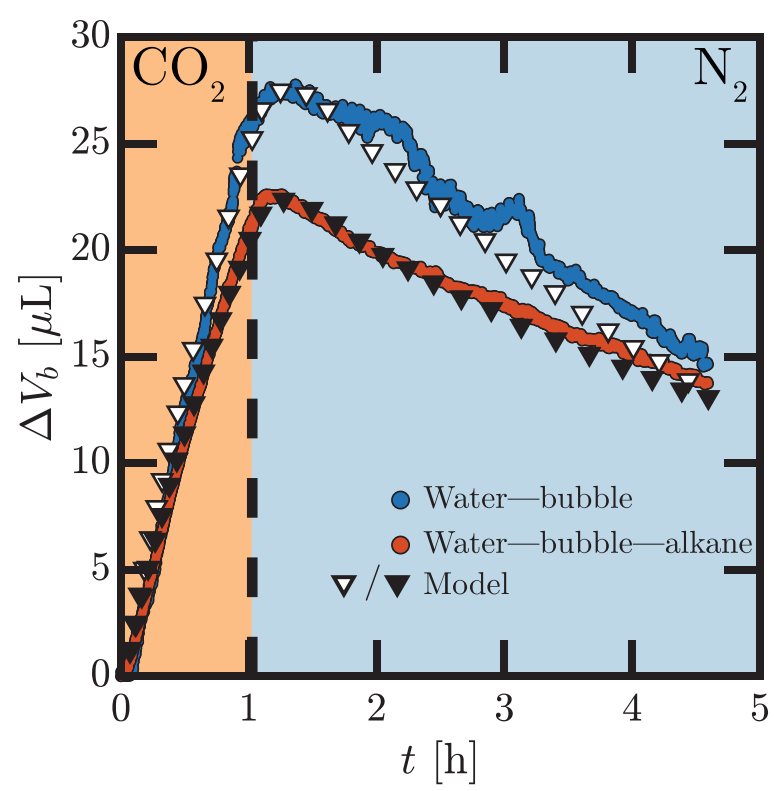

FIG. 9. Bubble growth dynamics for a binary and ternary system $\left(V_{f}=35 \mu \mathrm{L}, H_{w}=3 \mathrm{~mm}\right.$, and $H_{a}=$ $3 \mathrm{~mm}$ ) at $P_{0}=1$ bar. After $t=1 \mathrm{~h}$, the $\mathrm{CO}_{2}$ atmosphere inside the chamber is replaced by a $\mathrm{N}_{2}$ atmosphere at the same pressure, thereby enforcing dissolution.

the dissolution flux of air into the alkane is strongest at $t \approx t_{f}$. The onset of convective dissolution is as fast as the time required for the step in pressure to reach and stabilize at the experiment level [see Fig. 8(b) inset].

\section{B. Nitrogen solute exchange}

To further visualize the buffering effect of the hexadecane layer on bubble dissolution, a twocylinder experiment consisting of a binary and ternary system $\left(V_{f}=35 \mu \mathrm{L}, H_{w}=3 \mathrm{~mm}\right.$, and $H_{a}=3 \mathrm{~mm}$ ) was placed in a $\mathrm{CO}_{2}$ atmosphere at $P_{0}\left(t>t_{f}\right)=1.0$ bar. After $1 \mathrm{~h}$, the $\mathrm{CO}_{2}$ atmosphere is replaced by a $\mathrm{N}_{2}$ atmosphere at the same pressure. The resulting bubble dynamics are shown in Fig. 9. The first solute exchange event induces bubble growth, as discussed extensively in the previous sections. In contrast, the second solute exchange induces bubble dissolution, as $\mathrm{CO}_{2}$ from the bubbles redissolves back through the water barrier. Note that the opposing $\mathrm{N}_{2}$ flux is much weaker owing to its poor solubility in water. The buffering effect of the alkane layer stands out with the fact that the bubble in the ternary system shrinks more slowly compared to the bubble in the bubble-water system. This difference is caused by $\mathrm{CO}_{2}$ exsolving from the alkane back into the bubble. After replacing the $\mathrm{CO}_{2}$ atmosphere with $\mathrm{N}_{2}$, the $\mathrm{CO}_{2}$ concentration gradient across the water layer is effectively inverted. The dense $\mathrm{CO}_{2}$-rich boundary layer now rests at the bottom water-bubble interface. Stratification is stable thereon, and therefore the transport of $\mathrm{CO}_{2}$ can be assumed to be purely driven by diffusion. This evidently results in a slower dissolution rate. The model prediction importantly reflects the asymmetry between the growth and dissolution stages, and overlaps reasonably well with the experimental results.

\section{CONCLUSIONS AND OUTLOOK}

We have investigated the growth dynamics of a trapped slug bubble in a vertical glass cylinder beneath a short layer of water which separates the bubble from the ambient gas. Replacing the ambient air with a $\mathrm{CO}_{2}$ atmosphere induces an asymmetric exchange of the gaseous solutes between 
the $\mathrm{CO}_{2}$-rich water barrier and the air-rich bubble. Net bubble growth is always observed, even after pressurization. We refer to this process as solute exchange.

The dominant transport of $\mathrm{CO}_{2}$ across the water barrier is facilitated by diffusion and greatly enhanced by convective dissolution. Inverting the cylinder orientation has correspondingly been shown to suppress convection. The transport of $\mathrm{CO}_{2}$ across the layer was then fully driven by diffusion, resulting in slower growth. The bubble growth dynamics and underlying mass transport processes were characterized by means of a numerical model based on one-dimensional diffusive transport. Analytical solutions able to accurately predict the growth dynamics were subsequently derived. The effect of convective dissolution across the water layer was treated as a reduction of the effective diffusion length, in accordance with the expected scaling for laminar or natural convection. The fact that the Sherwood number scales to the $1 / 4$ power of the Rayleigh number was verified experimentally, and explains why the bubble growth rate was observed to increase with the ambient pressure but behaves inversely with the the water layer height.

Finally, the binary water-bubble system was compared to a ternary water-bubble-alkane system. The alkane ( $n$-hexadecane) layer was shown to act essentially as an unsteady sink (or source) of $\mathrm{CO}_{2}$ gas. As a result, the alkane layer induces a buffering effect on the bubble growth (or dissolution) dynamics. The slower growth curves were well reproduced by an extension of the numerical model and ultimately a single dynamical equation for the bubble volume derived under a set of simplifying assumptions.

Our findings offer insight on mass-transfer effects in microfluidic or microreactor devices comprising segmented gas-liquid phases or density-changing solutes, and also on the growth and elimination of trapped bubbles. Moreover, the differences in the growth rates between binary systems, ternary systems, or a combination thereof can offer a feasible means by which the physical properties of gases in the liquid layers can be obtained.

\section{ACKNOWLEDGMENTS}

This work was supported by the Netherlands Center for Multiscale Catalytic Energy Conversion (MCEC), an NWO Gravitation programme funded by the Ministry of Education, Culture and Science of the government of the Netherlands.

\section{APPENDIX A: DIFFUSION ACROSS A SHEET OF FINITE LENGTH}

Consider the canonical problem of one-dimensional diffusion across a sheet of finite length. One end of the sheet is kept at a constant surface concentration while the remaining end is at zero concentration. The sheet concentration profile is initially zero. With proper normalization, the dimensionless problem considers a surface concentration, diffusivity, and sheet length of unity. Our particular case involves the diffusion of $C_{\mathrm{CO}_{2}}^{w}\left(z, t>t_{f}\right)$ across a water layer of height $H_{w}$ with surface concentrations $C_{\mathrm{CO}_{2}}^{w}\left(H_{a}, t\right)=S_{\mathrm{CO}_{2}}^{w} P_{0}$ and $C_{\mathrm{CO}_{2}}^{w}(0, t)=0$. We then consider $c=C_{\mathrm{CO}_{2}}^{w} / S_{\mathrm{CO}_{2}}^{w} P_{0}$ as a function of $\xi=1-z / H_{w}$ and $\tau=D_{\mathrm{CO}_{2}}^{w}\left(t-t_{f}\right) / H_{w}^{2}$.

The solution to $\partial_{\tau} c=\partial_{\xi \xi} c$ subject to $c(0, \tau)=1, c(1, \tau)=0$, and $c(\xi, 0)=0$ is (see, e.g., Ref. [66])

$$
c(\xi, \tau)=(1-\xi)-\frac{2}{\pi} \sum_{n=1}^{\infty} \frac{1}{n} \sin (n \pi \xi) e^{-n^{2} \pi^{2} \tau} .
$$

Fluxes can be easily deduced from Eq. (A1). At any given time, the diffusion flux out of the sheet (across the zero-concentration end) is $-\left.\partial_{\xi} c(\xi, \tau)\right|_{\xi=1}=1-f^{\prime}(\tau)$, where

$$
f^{\prime}(\tau)=-2 \sum_{n=1}^{\infty}(-1)^{n} e^{-n^{2} \pi^{2} \tau}
$$


(a)

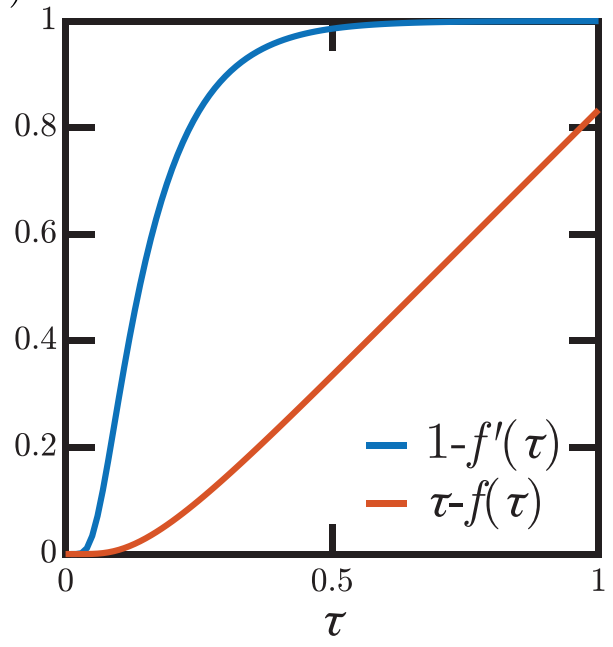

(b)

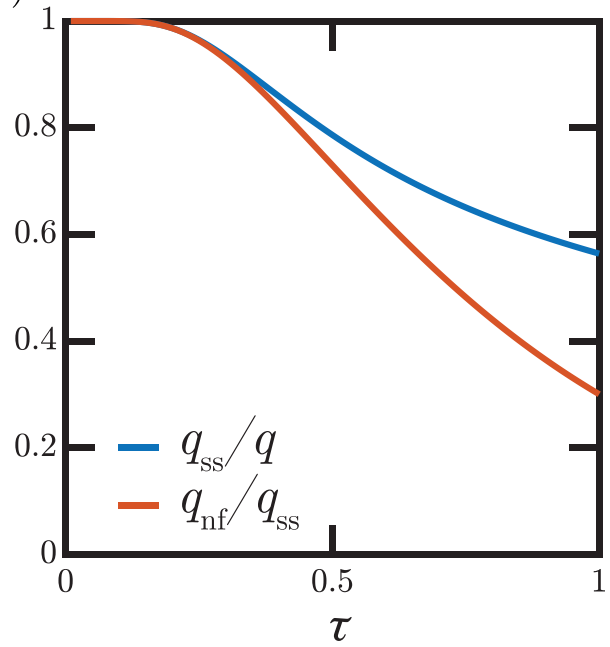

FIG. 10. (a) Diffusion outflux (blue line) and its time integral (red line) as functions of time. The transient period can be thought to last $\tau \sim 0.4$, after which the flux overcomes the $95 \%$ mark of the steady-state value. (b) Influx ratios: $q_{s s}$ denotes the (self-similar) diffusion influx into the unit-concentration boundary of an infinite sheet, $q$ is the influx into the unit-concentration boundary of a sheet of unit length delimited by a zero-concentration boundary, and $q_{n f}$ is the influx into the unit-concentration boundary of a sheet of unit length delimited by a no-flux boundary. In both cases, self-similarity breaks down after a time $\tau \sim 0.3$, where the flux ratios drop below $95 \%$.

is referred to as the transient flux function. It has limits $f^{\prime}(\infty)=0$ and $f^{\prime}(\tau \rightarrow 0)=1$ but $f^{\prime}(0)$ is nonanalytical in $\tau=0$. The diffusion flux $1-f^{\prime}(\tau)$ is plotted in Fig. 10(a) and it is equivalent to our Sherwood number given that it precisely quantifies mass transfer out of the sheet. This result is directly used in Eq. (12).

Figure 10(a) also plots the total amount of substance that has diffused out in time $\tau$, namely, $\tau-f(\tau)$, where

$$
f(\tau) \equiv \frac{1}{6}+\frac{2}{\pi^{2}} \sum_{n=1}^{\infty} \frac{(-1)^{n}}{n^{2}} e^{-n^{2} \pi^{2} \tau}
$$

is referred to as the transient flux integral function. It has limits $f(0)=0$, and $f(\infty)=1 / 6$.

In the limit $\tau \rightarrow 0$, Eq. (A1) converges to the self-similar solution $c_{s s}(\xi, \tau)=\operatorname{erfc}(\xi / \sqrt{4 \tau})$. The time at which self-similarity is broken can be estimated as the time at which the finite length of the sheet causes the influx (on $\xi=0$ ) to deviate from the self-similar solution by an arbitrary fractional amount. This is done in Fig. 10(b), which plots the ratio of the influx of the self-similar solution, $q_{s s}=1 / \sqrt{\pi \tau}$, to the influx of the solution in Eq. (A1), namely,

$$
q=1+2 \sum_{n=1}^{\infty} e^{-n^{2} \pi^{2} \tau}
$$

This result is relevant in the description of the diffusive transport in the water layer. In the case of the alkane layer, we should consider the diffusion across an equivalent sheet where the zeroconcentration boundary $c(1, \tau)=0$ is replaced by a no-flux boundary, i.e., $\partial c / \partial \xi=0$ on $\xi=1$. 
The influx (on $\xi=0$ ) becomes (see, e.g., Ref. [66])

$$
q_{n f}=2 \sum_{n=0}^{\infty} \exp \left[-\pi^{2}\left(n+\frac{1}{2}\right)^{2} \tau\right]
$$

Similarly, the time at which self-similarity is broken can be estimated as the time in which $q_{n f} / q_{s s} \leqslant$ 1 falls below an arbitrary threshold [see Fig. 10(b)].

\section{APPENDIX B: ONE-DIMENSIONAL NUMERICAL MODEL}

Following the notation of main text, the quantities associated with the water phase are indicated by subscript or superscript $w$; subscript or superscript $b$ refers to the bubble, $a$ to the alkane, and 0 to the ambient. The transport of molar concentration of gas species $j=\mathrm{CO}_{2}, \mathrm{O}_{2}$, or $\mathrm{N}_{2}$ dissolved in the water layer is modeled by the one-dimensional (1D) diffusion equation

$$
\frac{\partial C_{j}^{w}}{\partial t}=D_{j}^{w} \frac{\partial^{2} C_{j}^{w}}{\partial x^{2}}, \quad \text { on } \quad 0<x<H_{w} .
$$

The concentration boundary conditions in the water phase follow Henry's law,

$$
C_{j}^{w}(0, t)=S_{j}^{w} P_{j}^{b}(t), \quad C_{j}^{w}\left(H_{w}, t\right)=S_{j}^{w} X_{j}^{0}(t) P_{0}(t),
$$

where the ambient pressure $P_{0}(t)$ and ambient mole fractions $X_{j}^{0}(t)$ are prescribed at all times. In our case, the pressure history $P_{0}(t)$ is directly interpolated from the experimental measurements. In the presence of dissolution-driven convection, we must solve for $C_{\mathrm{CO}_{2}}^{w}$ within a single diffusive region (boundary layer) of length $\delta(t)$ as explained in the main text, that is,

$$
\frac{\partial C_{\mathrm{CO}_{2}}^{w}}{\partial t}=D_{\mathrm{CO}_{2}}^{w} \frac{\partial^{2} C_{\mathrm{CO}_{2}}^{w}}{\partial \xi^{2}} \text { on } 0<\xi<\delta(t) .
$$

The numerical difficulties inherent in solving the moving-boundary problem posed in Eq. (B3) can be circumvented by introducing a coordinate transformation $x(t)=H_{w} \xi / 2 \delta(t)$ which reverts the spatial domain back to $0 \leqslant x \leqslant H_{w}$. We then solve for $C_{\mathrm{CO}_{2}}^{w}(x, t)$ from

$$
\frac{\partial C_{\mathrm{CO}_{2}}^{w}}{\partial t}-\frac{\dot{\delta}}{\delta} x \frac{\partial C_{\mathrm{CO}_{2}}^{w}}{\partial x}=D_{\mathrm{CO}_{2}}^{w}\left(\frac{H_{w}}{\delta}\right)^{2} \frac{\partial^{2} C_{\mathrm{CO}_{2}}^{w}}{\partial x^{2}} \quad \text { on } \quad 0<x<H_{w} .
$$

The boundary conditions in Eq. (B2) therefore still apply. Note that a numerical advection term [second term on the left-hand side of Eq. (B4)] formally appears as a consequence of the coordinate $x(t)$ changing with time. It is, however, neglected under the assumption that the variations in $\delta$ are slow as compared to diffusion, i.e., $\delta \delta / D_{\mathrm{CO}_{2}}^{w} \ll 1$. The thickness $\delta$ is computed at every time step as established by the relationship in Eq. (5), where the numerical value of $\operatorname{Ra}_{w}(t)$ [as defined in Eq. (3)] is evaluated at the previous time step.

When modeling the ternary water-bubble-alkane system, we must additionally solve the diffusion equation in the alkane layer,

$$
\frac{\partial C_{j}^{a}}{\partial t}=D_{j}^{a} \frac{\partial^{2} C_{j}^{a}}{\partial y^{2}}, \quad \text { on } \quad 0<y<H_{a}
$$

with boundary conditions

$$
\frac{\partial C_{j}^{a}}{\partial y}(0, t)=0, \quad C_{j}^{a}\left(H_{a}, t\right)=S_{j}^{a} P_{j}^{b}(t) .
$$

The partial pressures inside the bubble are assumed proportional to the mole fractions, $P_{j}^{b}=$ $n_{j}^{b} P_{b} / n_{b}$, where $n_{b}(t)=\sum_{j} n_{j}^{b}(t)$ is the total number of moles of gas and the assumption $P_{b}(t)=$ 
$P_{0}(t)$ neglects the Laplace pressure in the bubble. The gas content in the bubble is found by integrating in time the molar fluxes into the bubble:

$$
\frac{4}{\pi d^{2}} \frac{\mathrm{d} n_{j}^{b}}{\mathrm{~d} t}=D_{j}^{w} \frac{\partial C_{j}^{w}}{\partial x}(x=0, t)-D_{j}^{a} \frac{\partial C_{j}^{a}}{\partial y}\left(y=H_{a}, t\right) .
$$

Finally, the bubble volume is assumed to evolve according to the ideal gas law, $P_{0}(t) V_{b}(t)=$ $n_{b}(t) R T_{0}$. Before flushing $\left(t<t_{f-\text { start }}\right)$, the system is in thermodynamic equilibrium. The initial bubble volume is $V_{b}\left(t<t_{f-\text { start }}\right)=V_{\text {eq }}$ and $P_{0}\left(t<t_{f-\text { start }}\right)=P_{\text {eq }}$. The initial conditions read

$$
\begin{aligned}
& C_{j}^{a}\left(x, t<t_{f-\text { start }}\right)=S_{j}^{a} P_{\mathrm{eq}} X_{j}^{0, \mathrm{eq}}, \\
& C_{j}^{w}\left(y, t<t_{f-\text { start }}\right)=S_{j}^{w} X_{j}^{0, \mathrm{eq}} P_{\mathrm{eq}},
\end{aligned}
$$

and

$$
n_{j}^{b}\left(t<t_{f-\text { start }}\right)=\frac{P_{\mathrm{eq}} X_{j}^{0, \mathrm{eq}} V_{\mathrm{eq}}}{R T_{0}},
$$

where $X_{\mathrm{CO}_{2}}^{0, \text { eq }}=0, X_{\mathrm{N}_{2}}^{0, \text { eq }}=0.79$, and $X_{\mathrm{O}_{2}}^{0 \text {,eq }}=0.21$ is the composition of dry air. Immediately upon flushing $\left(t>t_{f \text {-start }}\right)$, the ambient composition is set to reflect a pure $\mathrm{CO}_{2}$ atmosphere: $X_{\mathrm{CO}_{2}}^{0}=$ 1 , and $X_{N_{2}}^{0}=X_{O_{2}}^{0}=0$. The diffusion equations are discretized using a second-order central finitedifferences scheme. The resulting ODE system for $C_{j}^{w}(x, t), C_{j}^{a}(y, t)$, and $n_{j}^{b}(t)$ is integrated in time with a standard Runge-Kutta ODE solver.

[1] L. Jiang, Z. Xue, and H. Park, Enhancement of $\mathrm{CO}_{2}$ dissolution and sweep efficiency in saline aquifer by microbubble $\mathrm{CO}_{2}$ injection, Int. J. Heat Mass Transf. 138, 1211 (2019).

[2] H. E. Huppert and J. A. Neufeld, The fluid mechanics of carbon dioxide sequestration, Annu. Rev. Fluid Mech. 46, 255 (2014).

[3] J. B. Grotberg, Respiratory fluid mechanics, Phys. Fluids 23, 021301 (2011).

[4] Y. Cao, C. Soares, N. Padoin, and T. Noël, Gas bubbles have controversial effects on Taylor flow electrochemistry, Chem. Eng. J. 406, 126811 (2021).

[5] M. Abolhasani, A. Günther, and E. Kumacheva, Microfluidic studies of carbon dioxide, Angew. Chem. Int. Ed. 53, 7992 (2014).

[6] T. Cubaud, M. Sauzade, and R. Sun, $\mathrm{CO}_{2}$ dissolution in water using long serpentine microchannels, Biomicrofluidics 6, 022002 (2012).

[7] T. Lu, R. Fan, L. F. Delgadillo, and J. Wan, Stabilization of carbon dioxide $\left(\mathrm{CO}_{2}\right)$ bubbles in micrometerdiameter aqueous droplets and the formation of hollow microparticles, Lab Chip 16, 1587 (2016).

[8] E. Stride and M. Edirisinghe, Novel microbubble preparation technologies, Soft Matter 4, 2350 (2008).

[9] I. Pereiro, A. F. Khartchenko, L. Petrini, and G. V. Kaigala, Nip the bubble in the bud: A guide to avoid gas nucleation in microfluidics, Lab Chip 19, 2296 (2019).

[10] A. Volk, M. Rossi, C. J. Kähler, S. Hilgenfeldt, and A. Marin, Growth control of sessile microbubbles in PDMS devices, Lab Chip 15, 4607 (2015).

[11] R. B. Espinosa, M. H. Duits, D. Wijnperlé, F. Mugele, and L. Lefferts, Bubble formation in catalyst pores; curse or blessing? React. Chem. Eng. 3, 826 (2018).

[12] S. Prakash, W. Mustain, and P. A. Kohl, Carbon dioxide vent for direct methanol fuel cells, J. Power Sources 185, 392 (2008).

[13] D. D. Meng, T. Cubaud, C. Ho, and C. Kim, A methanol-tolerant gas-venting microchannel for a microdirect methanol fuel cell, J. Microelectromech. Syst. 16, 1403 (2007).

[14] C. Litterst, S. Eccarius, C. Hebling, R. Zengerle, and P. Koltay, Increasing $\mu$ DMFC efficiency by passive $\mathrm{CO}_{2}$ bubble removal and discontinuous operation, J. Micromech. Microeng. 16, S248 (2006). 
[15] N. Pande, S. K. Chandrasekar, D. Lohse, G. Mul, J. A. Wood, B. T. Mei, and D. Krug, Electrochemically induced $\mathrm{pH}$ change: Time-resolved confocal fluorescence microscopy measurements and comparison with numerical model, J. Phys. Chem. Lett. 11, 7042 (2020).

[16] D. Lohse, Fundamental fluid dynamics challenges in inkjet printing, Annu. Rev. Fluid Mech. 54, 1 (2022).

[17] D. Lohse, Bubble puzzles: From fundamentals to applications, Phys. Rev. Fluids 3, 110504 (2018).

[18] S. Peng, T. L. Mega, and X. Zhang, Collective effects in microbubble growth by solvent exchange, Langmuir 32, 11265 (2016).

[19] Z. Lu, H. Xu, H. Zeng, and X. Zhang, Solvent effects on the formation of surface nanodroplets by solvent exchange, Langmuir 31, 12120 (2015).

[20] M. C. Rogers and S. W. Morris, Buoyant Plumes and Vortex Rings in an Autocatalytic Chemical Reaction, Phys. Rev. Lett. 95, 024505 (2005).

[21] V. Loodts, H. Saghou, B. Knaepen, L. Rongy, and A. De Wit, Differential diffusivity effects in reactive convective dissolution, Fluids 3, 83 (2018).

[22] E. Dietrich, S. Wildeman, C. W. Visser, K. Hofhuis, E. S. Kooij, H. J. W. Zandvliet, and D. Lohse, Role of natural convection in the dissolution of sessile droplets, J. Fluid Mech. 794, 45 (2016).

[23] K. L. Chong, Y. Li, C. S. Ng, R. Verzicco, and D. Lohse, Convection-dominated dissolution for single and multiple immersed sessile droplets, J. Fluid Mech. 892, A21 (2020).

[24] Á. Moreno Soto, O. R. Enríquez, A. Prosperetti, D. Lohse, and D. van der Meer, Transition to convection in single bubble diffusive growth, J. Fluid Mech. 871, 332 (2019).

[25] G. Ahlers, S. Grossmann, and D. Lohse, Heat transfer and large scale dynamics in turbulent RayleighBénard convection, Rev. Mod. Phys. 81, 503 (2009).

[26] C. Yao, Y. Liu, S. Zhao, Z. Dong, and G. Chen, Bubble/droplet formation and mass transfer during gasliquid-liquid segmented flow with soluble gas in a microchannel, AIChE J. 63, 1727 (2017).

[27] K. Sklodowska and S. Jakiela, Enhancement of bacterial growth with the help of immiscible oxygenated oils, RSC Adv. 7, 40990 (2017).

[28] R. Sander, Compilation of Henry's law constants (version 4.0) for water as solvent, Atmos. Chem. Phys. 15, 4399 (2015).

[29] F. L. Smith and A. H. Harvey, Avoid common pitfalls when using Henry’s law, Chem. Eng. Prog. 103, 33 (2007).

[30] J. Makranczy, K. M. Megyery-Balog, L. Rusz, and L. Patyi, Solubility of gases in normal-alkanes, Hung. J. Ind. Chem. 4, 269 (1976).

[31] O. R. Enríquez, C. Hummelink, G.-W. Bruggert, D. Lohse, A. Prosperetti, D. van der Meer, and C. Sun, Growing bubbles in a slightly supersaturated liquid solution, Rev. Sci. Instrum. 84, 065111 (2013).

[32] B.-S. Chun and G. T. Wilkinson, Interfacial tension in high-pressure carbon dioxide mixtures, Ind. Eng. Chem. Res. 34, 4371 (1995).

[33] A. Tamimi, E. B. Rinker, and O. C. Sandall, Diffusion coefficients for hydrogen sulfide, carbon dioxide, and nitrous oxide in water over the temperature range 293-368 K, J. Chem. Eng. Data 39, 330 (1994).

[34] J. B. Matthews, M. A. Rodden, and A. Akgerman, High-temperature diffusion, viscosity, and density measurements in $n$-hexadecane, J. Chem. Eng. Data 32, 317 (1987).

[35] I. Zandi and C. D. Turner, The absorption of oxygen by dilute polymeric solutions molecular diffusivity measurements, Chem. Eng. Sci. 25, 517 (1970).

[36] L.-K. Ju and C. S. Ho, Oxygen diffusion coefficient and solubility in n-hexadecane, Biotechnol. Bioeng. 34, 1221 (1989).

[37] D. M. Himmelblau, Diffusion of dissolved gases in liquids, Chem. Rev. 64, 527 (1964).

[38] S. Backhaus, K. Turitsyn, and R. E. Ecke, Convective Instability and Mass Transport of Diffusion Layers in a Hele-Shaw Geometry, Phys. Rev. Lett. 106, 104501 (2011).

[39] B. Arendt, D. Dittmar, and R. Eggers, Interaction of interfacial convection and mass transfer effects in the system $\mathrm{CO}_{2}$-water, Int. J. Heat Mass Transf. 47, 3649 (2004).

[40] A. C. Slim, M. M. Bandi, J. C. Miller, and L. Mahadevan, Dissolution-driven convection in a Hele-Shaw cell, Phys. Fluids 25, 024101 (2013). 
[41] R. Farajzadeh, H. Salimi, P. L. J. Zitha, and H. Bruining, Numerical simulation of density-driven natural convection in porous media with application for $\mathrm{CO}_{2}$ injection projects, Int. J. Heat Mass Transf. 50, 5054 (2007).

[42] V. Loodts, L. Rongy, and A. De Wit, Impact of pressure, salt concentration, and temperature on the convective dissolution of carbon dioxide in aqueous solutions, Chaos 24, 043120 (2014).

[43] J. E. Garcia, Density of aqueous solutions of $\mathrm{CO}_{2}$, Lawrence Berkeley National Laboratory Technical Report (2001).

[44] S. Grossmann and D. Lohse, Scaling in thermal convection: A unifying theory, J. Fluid Mech. 407, 27 (2000).

[45] A. Bejan, Convection Heat Transfer (Wiley, New York, 2013).

[46] T. J. Kneafsey and K. Pruess, Laboratory experiments and numerical simulation studies of convectively enhanced carbon dioxide dissolution, Energy Procedia 4, 5114 (2011).

[47] F. Hébert, R. Hufschmid, J. Scheel, and G. Ahlers, Onset of Rayleigh-Bénard convection in cylindrical containers, Phys. Rev. E 81, 046318 (2010).

[48] W. V. R. Malkus, Discrete transitions in turbulent convection, Proc. Math. Phys. Eng. Sci. 225, 185 (1954).

[49] R. J. Goldstein, E. M. Sparrow, and D. C. Jones, Natural convection mass transfer adjacent to horizontal plates, Int. J. Heat Mass Transf. 16, 1025 (1973).

[50] J. R. Lloyd and W. R. Moran, Natural convection adjacent to horizontal surface of various planforms, J. Heat Transfer 96, 443 (1974).

[51] G. O. Roberts, Fast viscous Bénard convection, Geophys. Astrophys. Fluid Dyn. 12, 235 (1979).

[52] M. Vynnycky and Y. Masuda, Rayleigh-Bénard convection at high Rayleigh number and infinite Prandtl number: Asymptotics and numerics, Phys. Fluids 25, 113602 (2013).

[53] S. Grossmann and D. Lohse, Thermal Convection for Large Prandtl Numbers, Phys. Rev. Lett. 86, 3316 (2001).

[54] J. Jimenez and J. A. Zufiria, A boundary-layer analysis of Rayleigh-Bénard convection at large Rayleigh number, J. Fluid Mech. 178, 53 (1987).

[55] A. Maali, R. Boisgard, H. Chraibi, Z. Zhang, H. Kellay, and A. Würger, Viscoelastic Drag Forces and Crossover from No-Slip to Slip Boundary Conditions for Flow near Air-Water Interfaces, Phys. Rev. Lett. 118, 084501 (2017).

[56] L. N. Howard, Convection at high Rayleigh number, in Applied Mechanics, edited by H. Görtler (Springer, Berlin, 1966), pp. 1109-1115.

[57] M. V. R. Malkus, The heat transport and spectrum of thermal turbulence, Proc. R. Soc. London A 225, 196 (1954).

[58] Y. Song, M. Hao, Y. Liu, Y. Zhao, B. Su, and L. Jiang, $\mathrm{CO}_{2}$ diffusion in n-hexadecane investigated using magnetic resonance imaging and pressure decay measurements, RSC Adv. 4, 50180 (2014).

[59] P. Yang, H. Guo, Z. Wang, and Q. Zhou, Density and volumetric properties of binary mixtures of $\mathrm{CO}_{2}+$ hexadecane from (303.2 to 473.2) K and pressures up to 50.0 MPa, J. Chem. Eng. Data 64, 2568 (2019).

[60] M. McBride-Wright, G. C. Maitland, and J. P. M. Trusler, Viscosity and density of aqueous solutions of carbon dioxide at temperatures from (274 to 449$) \mathrm{K}$ and at pressures up to $100 \mathrm{MPa}$, J. Chem. Eng. Data 60, 171 (2015).

[61] H. Watanabe and K. Iizuka, The influence of dissolved gases on the density of water, Metrologia 21, 19 (1985).

[62] H. S. Carslaw and J. C. Jaeger, Conduction of Heat in Solids (Clarendon Press, Oxford, UK, 1959).

[63] L. D. Landau and E. M. Lifshitz, Thermal conduction in fluids, inFluid Mechanics, 2nd ed. (Pergamon, New York, 1987), Chap. 5, p. 200.

[64] P. Peñas-López, M. A. Parrales, J. Rodríguez-Rodríguez, and D. van der Meer, The history effect in bubble growth and dissolution. Part 1. Theory, J. Fluid Mech. 800, 180 (2016).

[65] S. Chu and A. Prosperetti, Dissolution and growth of a multicomponent drop in an immiscible liquid, J. Fluid Mech., 798, 787 (2016).

[66] J. Crank, in The Mathematics of Diffusion, 2nd ed. (Oxford University Press, Oxford, UK, 1975), pp. 49-52. 